\title{
Fuzzy EOQ Model for Time Varying Deterioration and Exponential Time Dependent Demand Rate under Inflation
}

\author{
K.Geetha*, S.P.Reshma \\ Department of Mathematics, Emerald Heights College for Women, Udagamandalam, The Nilgiris, Tamil Nadu, India
}

\begin{abstract}
Received September 8, 2021; Revised January 15, 2022; Accepted January 25, 2022
Cite This Paper in the following Citation Styles

(a): [1] K.Geetha1, S.P.Reshma, "Fuzzy EOQ Model for Time Varying Deterioration and Exponential Time Dependent Demand Rate under Inflation," Mathematics and Statistics, Vol.10, No.1, pp. 251-261, 2022. DOI: 10.13189/ms.2022.100124

(b): K.Geethal, S.P.Reshma, (2022). Fuzzy EOQ Model for Time Varying Deterioration and Exponential Time Dependent Demand Rate under Inflation. Mathematics and Statistics, 10(1), 251-261. DOI: 10.13189/ms.2022.100124
\end{abstract}

Copyright $@ 2022$ by authors, all rights reserved. Authors agree that this article remains permanently open access under the terms of the Creative Commons Attribution License 4.0 International License

\begin{abstract}
In this study, we have discussed a fuzzy eoq model for deteriorating products with time varying deterioration under inflation and exponential time dependent demand rate. Shortages are not allowed in this fuzzy eoq model and the impact of inflation is investigated. An inventory model is used to determine whether the order quantity is more than or equal to a predetermined quantity for declining items. The optimal solution for the existing model is derived by taking truncated taylor's series approximation for finding closed form optimal solution. The cost of deterioration, cost of ordering , cost of holding and the time taken to settle the delay in account are considered using triangular fuzzy numbers. In this study, the fuzzy triangular numbers are used to estimate the optimal order quantity and cycle duration. Furthermore, we have used graded mean integration method and signed distance approach to defuzzify these values. To validate our model, numerical examples are discussed for all cases with the help of sensitivity analysis for different parameters. Finally, a higher decay rate results in a shorter ideal cycle time as well as higher overall relevant cost is established. The presented model can be used to predict demand as a quadratic function of time,stock level time dependent demand, selling price, and other variables.
\end{abstract}

Keywords Inventory, Inflation, Exponential Time Dependent, Triangular Fuzzy Number, Defuzzification

\section{Introduction}

An inventory control systems principal goal is to determine when and how much to order.Inventory management is complicated by deterioration. Everything deteriorates over time. Deterioration might be gradual or rapid, thus it's crucial to factor it into your EOQ model. Many scholars are interested in building trade credit-based mathematical models. Goyal, who looked at inventory models with a pre-authorized payment delay. According to numerous research, the cost does not alter over the planning horizon. This assertion may not be accurate, despite the fact that many countries have significant inflation rates. Inflation has an impact on the demand for particular goods. As the value of money decreases, the inflation rate rises. As a result, when selecting the best inventory policy, the impact of inflation and the time worth of money cannot be overlooked.

Dutta and Kumar [1] used a fuzzy trapezoidal number and the Signed distance method to create a fuzzy inventory model without shortages. Jaggi et al [8] created a fuzzy inventory model with deterioration that took demand into account as time-varying. Kumar and Rajput [12] proposed a fuzzy inventory model for deteriorating products with time-dependent demand and partial backlog for deteriorating items with timedependent demand and partial backlog. Economic order quantity in Fuzzy sense for Inventory without backorder Fuzzy sets was developed by Huey -Ming Lee and Jing-shing [6]. Harish Nagar and Priyanka Surana [5] developed a fuzzy inventory model for deteriorating objects that used pentagonal fuzzy numbers as parameters. In a fuzzy world, Dutta and Pavan Kumar[2] built an inventory model without shortages by taking into account keeping costs, ordering costs, and demand. Jershan Chiang-Shing Yao and Huey-Ming Lee [9] deal with 
backordered inventory. S.K.Indirajit Sinha, P.N.Samantha, and U.K.Mishra [7] use the signed distance approach to solve a fuzzy inventory model of shortages under completely backlogged conditions. Jershan Chiang-Shing Yao and Huey-Ming Lee [9] deal with backordered inventory. S.K.Indirajit Sinha, P.N.Samantha, and U.K.Mishra [7] use the signed distance approach to solve a fuzzy inventory model of shortages under completely backlogged conditions. G.Michael Rosario and R.M.Rajalakshmi [13] used different fuzzy numbers and defuzzified using the signed distance approach to examine an inventory model of allowable shortage. As supplier credit is related to order quantity, Tripathi, R.P., and Mishra, T. [19] developed an eoq model with exponential time-dependent demand rate under inflation.

In this paper an fuzzy eoq model for deteriorating products with time varying deterioration under inflation and exponential time dependent demand rate is adopted for consideration. The main objective is to estimate the optimal order quantity and optimal cycle time using triangular fuzzy numbers. Further more for defuzzification these quantities we use graded mean integration and signed distance method.Finally the model is illustrated by numerical example.

\section{Notations and Assumptions}

This paper makes use of the following notations:

$\tilde{h}$ : fuzzy keeping cost rate per unit time

$r$ : inflation rate is stable over time., where $0 \leq r<1$

$p e^{r t}$ : selling price per unit at time, where $p$ is the unit selling price at time zero.

$\tilde{C} e^{r t}$ : fuzzy purchase cost per unit at time $t$, where $c$ is the unit purchase cost at time zero and $p>c$

$\tilde{A} e^{r t}$ : fuzzy ordering cost per order at time $t$

$H$ : length of planning horizon

$\tilde{m}$ : the maximum amount of time that can be allowed before settling an account

$I_{c}:$ Interest paid per $\$$ in stock per year

$I_{d}:$ Interest gained per unit

$Q:$ order quantity

$Q_{d}$ : minimum order quantity for which payment delays are allowed

$T$ : interval between refills

$T_{d}$ : the time period due to time-dependent demand in which $Q_{d}$ units are depleted to zero.

$I(t)$ : stock at time $t$

$R(t)$ : fuzzy yearly demand as a $R(t)=\lambda e^{\alpha t}, \lambda>0,0<$ $\alpha=1$

$\tilde{Z}(T):$ fuzzy total relevant cost over $(0, H)$

\section{Assumptions}

1. The rate of inflation remains constant.

2. No shortages are permitted.

3. As time passes, the market for the item increases exponentially.

4. Instantaneous replenishment.
5.If $Q<Q_{d}$, payments for obtained goods must be produced as soon as possible..

6.If, $Q=Q_{d}$, a payment delay up to $m$ is allowed.

The generated sales revenue is deposited in an interestbearing account because the account is not settled within the allowed time frame.. The customer pays off all units bought at the end of the credit period and starts paying the interest on the products in stock.

\section{Mathematical formulation}

Assume that the horizon length is $H=n T$, with $\mathrm{n}$ indicating the amount of replenishments to be produced during the period $H$ and $T$ indicating the time interval between replenishments. To meet demand, the inventory level $I(t)$ is gradually reduced. As a consequence, the variance in inventory over time can be calculated as

$$
\frac{d I(t)}{d t}+\theta t I(t)=-\lambda e^{\alpha t}, 0 \leq t \leq T=\frac{H}{n} ; 0 \leq \alpha \leq 1
$$

With boundary conditions $I(0)=Q$ and $I(T)=0$. Solution of (1) is given by

$$
\begin{array}{r}
I(t)=\lambda\left[(T-t)+\frac{\alpha}{2}\left(T^{2}-t^{2}\right)+\frac{\theta}{6}\left(T^{3}-t^{3}\right)\right] e^{\frac{-\theta t^{2}}{2}}, \\
0 \leq t \leq T
\end{array}
$$

And order quantity is

$$
Q=\lambda\left[T+\frac{\alpha}{2} T^{2}+\frac{\theta}{6} T^{3}\right]
$$

Since the time interval have equal lengths, we have

$$
\begin{aligned}
I(k T+t) & =\lambda\left[(T-t)+\frac{\alpha}{2}\left(T^{2}-t^{2}\right)\right. \\
& \left.+\frac{\theta}{6}\left(T^{3}-t^{3}\right)\right] e^{\frac{-\theta t^{2}}{2}}, 0 \leq t \leq T
\end{aligned}
$$

By using the order quantity, we determine the time period during which $Q_{d}$ units are depleted to zero due to demand. Then we have

$$
T_{d}=\frac{Q_{d}}{\lambda\left[1+\frac{\alpha}{2} T_{d}+\frac{\theta}{6} T_{d}^{2}\right]} \text { (approximately) }
$$

To calculate the overall applicable cost in $[0, H]$, we add the ordering, buying, and keeping costs together.:

(a) $O C=\sum_{k=0}^{n-1} A(k T)=A\left(\frac{e^{r H}-1}{e^{r T}-1}\right)$

(b) Purchasing cost $P C=\sum_{k=0}^{n-1} I(0) C(k T)$

$$
=C \lambda\left[T+\frac{\alpha}{2} T^{2}+\frac{\theta}{6} T^{3}\right]\left(\frac{e^{r H}-1}{e^{r T}-1}\right)
$$

(c) $H C=h \sum_{k=0}^{n-1} C(k T) \int_{0}^{T} I(k T+t) d t$ 


$$
=h C \lambda\left[\frac{T^{2}}{2}+\frac{\alpha}{3} T^{3}+\frac{\theta}{12} T^{4}\right]\left(\frac{e^{r H}-1}{e^{r T}-1}\right)
$$

Case(i) $0<T<T_{d}$

Payment delays are not allowed in this case since the cycle time interval $T<T_{d}$. When the customer receives the goods, the supplier must be paid immediately. Because interest is paid on all unsold products from the beginning,

$$
\begin{aligned}
I C_{1} & =I_{c} \sum_{k=0}^{n-1} C(k T) \int_{0}^{T} I(k T+t) d t \\
& =I_{c} C \lambda\left[\frac{T^{2}}{2}+\frac{\alpha}{3} T^{3}+\frac{\theta}{12} T^{4}\right]\left(\frac{e^{r H}-1}{e^{r T}-1}\right) \\
Z_{1}(T) & =O C+P C+H C+I C C_{1} \\
& =\left[A+C \lambda\left[T+\frac{\alpha}{2} T^{2}+\frac{\theta}{6} T^{3}\right]+h C \lambda\left[\frac{T^{2}}{2}+\frac{\alpha}{3} T^{3}\right.\right. \\
& \left.\left.+\frac{\theta}{12} T^{4}\right]+I_{c} C \lambda\left[\frac{T^{2}}{2}+\frac{\alpha}{3} T^{3}+\frac{\theta}{12} T^{4}\right]\right]\left(\frac{e^{r H}-1}{e^{r T}-1}\right) \\
& =\left[\frac{A}{T}+C \lambda\left[1+\frac{\alpha T}{2}+\frac{\theta}{6} T^{2}\right]+\left(h+I_{c}\right) C \lambda\left[\frac{T}{2}\right.\right. \\
& \left.+\frac{\alpha}{3} T^{2}+\frac{\theta}{12} T^{3}\right]-\frac{A r}{2}-\frac{r C \lambda}{2}\left(T+\frac{\alpha}{2} T^{2}+\frac{\theta}{6} T^{3}\right) \\
-\left(h+I_{c}\right) & \left.\frac{C \lambda r}{2}\left[\frac{T^{2}}{2}+\frac{\alpha}{3} T^{3}+\frac{\theta}{12} T^{4}\right]\right]\left(H\left(1+\frac{r H}{2}\right)\right)
\end{aligned}
$$

Case(ii) $T_{d} \leq m<T$

Permissible delay $m$ occurs which is more than the interval between cycles $T$, since $T_{d} \leq m<T$. As a result, no interest is paid ie $I C_{2}=0$, but the interest received in $(0, H)$ is calculated as follows:

$$
\begin{aligned}
I E_{2}= & I_{d} \sum_{k=0}^{n-1} P(k T)\left(\int_{0}^{T} \lambda e^{\alpha t} t d t+(m-T) \int_{0}^{T} \lambda e^{\alpha t} d t\right) \\
& =\frac{I_{d} P \lambda}{\alpha}\left(\left(e^{\alpha T}-1\right)\left(m-\frac{1}{\alpha}\right)+T\right)\left(\frac{e^{r H}-1}{e^{r T}-1}\right) \quad(11) \\
Z_{2}(T) & =O C+P C+H C-I E_{2} \\
& =\left[\frac{A}{T}+C \lambda\left[1+\frac{\alpha T}{2}+\frac{\theta}{6} T^{2}\right]+h C \lambda\left[\frac{T}{2}\right.\right. \\
& \left.+\frac{\alpha}{3} T^{2}+\frac{\theta}{12} T^{3}\right]-\frac{A r}{2}-\frac{r C \lambda}{2}\left(T+\frac{\alpha}{2} T^{2}\right. \\
& \left.+\frac{\theta}{6} T^{3}\right)-\frac{h C \lambda r}{2}\left[\frac{T^{2}}{2}+\frac{\alpha}{3} T^{3}+\frac{\theta}{12} T^{4}\right] \\
& -I_{d} P \lambda\left(m+\frac{T}{2}(m \alpha-1)\right)+\frac{I_{d} P \lambda r}{2}(m T \\
& \left.\left.+\frac{T^{2}}{2}(m \alpha-1)\right)\right]\left(H\left(1+\frac{r H}{2}\right)\right)
\end{aligned}
$$

Case(iii) $T_{d} \leq m \leq T$

Delays in payments are allowed since $T \geq m \geq T_{d}$, and the overall applicable expense includes both the interest paid and the interest received. In $(0, H)$, the interest rate is

$$
\begin{aligned}
I C_{3} & =I_{c} \sum_{k=0}^{n-1} C(k T)\left(\int_{m}^{T} I(k T+t) d t\right) \\
& =I_{c} C \lambda\left[\frac{T^{2}}{2}+\frac{\alpha}{3} T^{3}+\frac{\theta}{8} T^{4}-\frac{\theta}{24} T^{4}\right.
\end{aligned}
$$

$$
\begin{aligned}
& -\frac{m}{2}(2 T-m)-\frac{\alpha m}{6}\left(3 T^{2}-m^{2}\right) \\
& \left.-\frac{\theta m}{24}\left(4 T^{3}-4 T m^{2}+2 m^{3}\right)\right]\left(\frac{e^{r H}-1}{e^{r T}-1}\right)
\end{aligned}
$$

The interest gained in $[0, H]$ is given by

$$
\begin{aligned}
& I E_{3}=I_{d} \sum_{k=0}^{n-1} P(k T)\left(\int_{0}^{m} \lambda e^{\alpha t} t d t\right) \\
& =\frac{I_{d} P \lambda}{\alpha}\left(m e^{\alpha m}-\frac{1}{\alpha}\left(e^{\alpha m}-1\right)\right)\left(\frac{e^{r H}-1}{e^{r T}-1}\right) \\
& Z_{3}(T)=O C+P C+H C+I C_{3}-I E_{3} \\
& =\left[\frac{A}{T}+C \lambda\left[1+\frac{\alpha T}{2}+\frac{\theta}{6} T^{2}+\frac{h T}{2}+\frac{h \alpha}{3} T^{2}+\frac{h \theta}{12} T^{3}\right.\right. \\
& -\frac{r T}{2}-\frac{r \alpha}{4} T^{2}-\frac{r \theta}{12} T^{3}-\frac{h r T^{2}}{4} \\
& \left.-\frac{h r \alpha}{6} T^{3}-\frac{h r \theta}{24} T^{4}\right]+I_{c} C \lambda\left[\frac{T}{2}+\frac{\alpha}{3} T^{2}+\frac{\theta}{12} T^{3}\right. \\
& -m+\frac{m^{2}}{2 T}-\frac{\alpha m T}{2}+\frac{\alpha m^{3}}{6 T}-\frac{m \theta}{6} T^{2} \\
& +\frac{\theta m^{3}}{6}+\frac{\theta m^{4}}{12 T}-\frac{r}{4} T^{2}-\frac{r \alpha}{6} T^{3}-\frac{r \theta}{24} T^{4}+\frac{r m T}{2} \\
& -\frac{r m^{2}}{4}+\frac{r m \alpha T^{2}}{4}-\frac{r \alpha m^{3}}{12}+\frac{r m \theta}{12} T^{3}-\frac{r \theta m^{3}}{12} T \\
& \left.-\frac{r \theta m^{4}}{24}\right]-\frac{A r}{2}-\frac{I_{d} P \lambda}{\alpha T}\left(m^{2}(m \alpha+1)\right) \\
& \left.+\frac{I_{d} P \lambda r}{2 \alpha}\left(m^{2}(m \alpha+1)\right)\right]\left(H\left(1+\frac{r H}{2}\right)\right)
\end{aligned}
$$

Case (iv) $m \leq T_{d} \leq T$

Case (iv) is similar to case (iii), since $\mathrm{T} \geq T_{d} \geq \mathrm{m}$, As a result, in $(0, \mathrm{H})$, the total applicable cost is

$$
\begin{aligned}
& Z_{4}(T)=\left[\frac{A}{T}+C \lambda\left[1+\frac{\alpha T}{2}+\frac{\theta}{6} T^{2}+\frac{h T}{2}+\frac{h \alpha}{3} T^{2}\right.\right. \\
& +\frac{h \theta}{12} T^{3}-\frac{r T}{2}-\frac{r \alpha}{4} T^{2}-\frac{r \theta}{12} T^{3}-\frac{h r T^{2}}{4} \\
& \left.-\frac{h r \alpha}{6} T^{3}-\frac{h r \theta}{24} T^{4}\right]+I_{c} C \lambda\left[\frac{T}{2}+\frac{\alpha}{3} T^{2}+\frac{\theta}{12} T^{3}\right. \\
& -m+\frac{m^{2}}{2 T}-\frac{\alpha m T}{2}+\frac{\alpha m^{3}}{6 T}-\frac{m \theta}{6} T^{2} \\
& +\frac{\theta m^{3}}{6}+\frac{\theta m^{4}}{12 T}-\frac{r}{4} T^{2}-\frac{r \alpha}{6} T^{3}-\frac{r \theta}{24} T^{4}+\frac{r m T}{2} \\
& -\frac{r m^{2}}{4}+\frac{r m \alpha T^{2}}{4}-\frac{r \alpha m^{3}}{12}+\frac{r m \theta}{12} T^{3}-\frac{r \theta m^{3}}{12} T \\
& \left.-\frac{r \theta m^{4}}{24}\right]-\frac{A r}{2}-\frac{I_{d} P \lambda}{2 T}\left(m^{2}(m \alpha+1)\right) \\
& \left.+\frac{I_{d} P \lambda r}{2 \alpha}\left(m^{2}(m \alpha+1)\right)\right]\left(H\left(1+\frac{r H}{2}\right)\right)
\end{aligned}
$$

\section{Fuzzy Model:}

Let $\tilde{A}=\left(a_{1}, a_{2}, a_{3}\right), \tilde{C}=\left(C_{1}, C_{2}, C_{3}\right)$,

$\tilde{h}=\left(h_{1}, h_{2}, h_{3}\right), \tilde{m}=\left(m_{1}, m_{2}, m_{3}\right), \tilde{\alpha}=\left(\alpha_{1}, \alpha_{2}, \alpha_{3}\right)$,

$\tilde{\theta}=\left(\theta_{1}, \theta_{2}, \theta_{3}\right), \tilde{\lambda}=\left(\lambda_{1}, \lambda_{2}, \lambda_{3}\right)$.

The total cost per unit time in a fuzzy context is calculated using the above triangular fuzzy numbers. 
Case(i) $0<T<T_{d}$

$$
\begin{aligned}
& \widetilde{Z_{1}(T)}=\left[\frac{A}{T}+C \lambda\left[1+\frac{\alpha T}{2}+\frac{\theta}{6} T^{2}\right]+\left(h+I_{c}\right) C \lambda\left[\frac{T}{2}\right.\right. \\
& \left.+\frac{\alpha}{3} T^{2}+\frac{\theta}{12} T^{3}\right]-\frac{A r}{2}-\frac{r C \lambda}{2}\left(T+\frac{\alpha}{2} T^{2}+\frac{\theta}{6} T^{3}\right) \\
& \left.-\left(h+I_{c}\right) \frac{C \lambda r}{2}\left[\frac{T}{2}+\frac{\alpha}{3} T^{2}+\frac{\theta}{12} T^{3}\right]\right]\left(H\left(1+\frac{r H}{2}\right)\right)
\end{aligned}
$$

Now defuzzifing the total cost $\widetilde{Z_{1}(T)}$ by using Signed distance method, we have

$$
\begin{aligned}
& Z_{1(s d)}(T)=\frac{1}{4}\left\{Z_{1\left(s d_{1}\right)}(T)+2 \underset{Z_{1\left(s d_{2}\right)}}{\widetilde{C}}(T)+Z_{1\left(s d_{3}\right)}(T)\right\} \\
& =\left\{\left\{\frac { 1 } { 4 } \left[\frac{a_{1}}{T}+C_{1} \lambda_{1}\left[1+\frac{\alpha_{1} T}{2}+\frac{\theta_{1}}{6} T^{2}\right]+\left(h_{1}+I_{c}\right) C_{1} \lambda_{1}\right.\right.\right. \\
& \times\left[\frac{T}{2}+\frac{\alpha_{1}}{3} T^{2}+\frac{\theta_{1}}{12} T^{3}\right]-\frac{\alpha_{1} r}{2}-\frac{r C_{1} \lambda_{1}}{2}\left[T+\frac{\alpha_{1}}{2} T^{2}\right. \\
& \left.\left.\left.+\frac{\theta_{1}}{6} T^{3}\right]-\left(h_{1}+I_{c}\right) \frac{C_{1} \lambda_{1} r}{2}\left[\frac{T}{2}+\frac{\alpha_{1}}{3} T^{2}+\frac{\theta_{1}}{12} T^{3}\right]\right]\right\} \\
& +\left\{\frac { 1 } { 2 } \left[\frac{a_{2}}{T}+C_{2} \lambda_{2}\left[1+\frac{\alpha_{2} T}{2}+\frac{\theta_{2}}{6} T^{2}\right]+\left(h_{2}+I_{c}\right) C_{2} \lambda_{2}\right.\right. \\
& \times\left[\frac{T}{2}+\frac{\alpha_{2}}{3} T^{2}+\frac{\theta_{2}}{12} T^{3}\right]-\frac{\alpha_{2} r}{2}-\frac{r C_{2} \lambda_{2}}{2}\left[T+\frac{\alpha_{2}}{2} T^{2}\right. \\
& \left.\left.\left.+\frac{\theta_{2}}{6} T^{3}\right]-\left(h_{2}+I_{c}\right) \frac{C_{2} \lambda_{2} r}{2}\left[\frac{T}{2}+\frac{\alpha_{2}}{3} T^{2}+\frac{\theta_{2}}{12} T^{3}\right]\right]\right\} \\
& +\left\{\frac { 1 } { 4 } \left[\frac{a_{3}}{T}+C_{3} \lambda_{3}\left[1+\frac{\alpha_{3} T}{2}+\frac{\theta_{3}}{6} T^{2}\right]+\left(h_{3}+I_{c}\right) C_{3} \lambda_{3}\left[\frac{T}{2}\right.\right.\right. \\
& \left.+\frac{\alpha_{3}}{3} T^{2}+\frac{\theta_{3}}{12} T^{3}\right]-\frac{\alpha_{3} r}{2}-\frac{r C_{3} \lambda_{3}}{2}\left[T+\frac{\alpha_{3}}{2} T^{2}+\frac{\theta_{3}}{6} T^{3}\right] \\
& \left.\left.\left.-\left(h_{3}+I_{c}\right) \frac{C_{3} \lambda_{3} r}{2}\left[\frac{T}{2}+\frac{\alpha_{3}}{3} T^{2}+\frac{\theta_{3}}{12} T^{3}\right]\right]\right\}\right\}\left(H\left(1+\frac{r H}{2}\right)\right)
\end{aligned}
$$

The necessary condition for minimizing the total cost is $\frac{\partial Z_{1(s) l}(T)}{\partial T}=0$ provided that they satisfy the sufficient conditions $\frac{\partial^{2} Z_{1(s d)}(T)}{\partial T^{2}}>0$

$$
\begin{aligned}
& \left\{\left\{\frac { 1 } { 4 } \left[\frac{-a_{1}}{T^{2}}+C_{1} \lambda_{1}\left[\frac{\alpha_{1}}{2}+\frac{\theta_{1}}{3} T-\frac{r}{2}-\frac{r \alpha_{1}}{2} T+\frac{r \theta_{1}}{4} T^{2}\right]\right.\right.\right. \\
& +\left(h_{1}+I_{c}\right) C_{1} \lambda_{1}\left[\frac{1}{2}+\frac{2 \alpha_{1}}{3} T+\frac{\theta_{1}}{4} T^{2}-\frac{r T}{2}-\frac{r \alpha_{1}}{2} T^{2}\right. \\
& \left.\left.\left.-\frac{r \theta_{1}}{3} T^{3}\right]\right]\right\}+\left\{\frac { 1 } { 2 } \left[\frac{-a_{2}}{T^{2}}+C_{2} \lambda_{2}\left[\frac{\alpha_{2}}{2}+\frac{\theta_{2}}{3} T-\frac{r}{2}-\frac{r \alpha_{2}}{2} T\right.\right.\right. \\
& \left.+\frac{r \theta_{2}}{4} T^{2}\right]+\left(h_{2}+I_{c}\right) C_{2} \lambda_{2}\left[\frac{1}{2}+\frac{2 \alpha_{2}}{3} T+\frac{\theta_{2}}{4} T^{2}-\frac{r T}{2}\right. \\
& \left.\left.\left.-\frac{r \alpha_{2}}{2} T^{2}-\frac{r \theta_{2}}{3} T^{3}\right]\right]\right\}+\left\{\frac { 1 } { 4 } \left[\frac{-a_{3}}{T^{2}}+C_{3} \lambda_{3}\left[\frac{\alpha_{3}}{2}+\frac{\theta_{3}}{3} T-\frac{r}{2}\right.\right.\right. \\
& \left.-\frac{r \alpha_{3}}{2} T+\frac{r \theta_{3}}{4} T^{2}\right]+\left(h_{3}+I_{c}\right) C_{3} \lambda_{3}\left[\frac{1}{2}+\frac{2 \alpha_{3}}{3} T+\frac{\theta_{3}}{4} T^{2}\right. \\
& \left.\left.\left.\left.-\frac{r T}{2}-\frac{r \alpha_{3}}{2} T^{2}-\frac{r \theta_{3}}{3} T^{3}\right]\right]\right\}\right\}\left(H\left(1+\frac{r H}{2}\right)\right)=0
\end{aligned}
$$

Now defuzzifing the total cost $\widetilde{Z_{1}(T)}$ by using Graded mean integration method, we have

$$
\begin{aligned}
& Z_{1(g m)}(T)=\frac{1}{6}\left\{Z_{1\left(g m_{1}\right)}(T)+4 Z_{1\left(g m_{2}\right)}^{\sim}(T)+Z_{1\left(g m_{3}\right)}(T)\right\} \\
& =\left\{\left\{\frac { 1 } { 6 } \left[\frac{a_{1}}{T}+C_{1} \lambda_{1}\left[1+\frac{\alpha_{1} T}{2}+\frac{\theta_{1}}{6} T^{2}\right]\right.\right.\right.
\end{aligned}
$$

$$
\begin{aligned}
& +\left(h_{1}+I_{c}\right) C_{1} \lambda_{1}\left[\frac{T}{2}+\frac{\alpha_{1}}{3} T^{2}+\frac{\theta_{1}}{12} T^{3}\right]-\frac{\alpha_{1} r}{2} \\
& -\frac{r C_{1} \lambda_{1}}{2}\left[T+\frac{\alpha_{1}}{2} T^{2}+\frac{\theta_{1}}{6} T^{3}\right]-\left(h_{1}+I_{c}\right) \frac{C_{1} \lambda_{1} r}{2}\left[\frac{T}{2}\right. \\
& \left.\left.\left.+\frac{\alpha_{1}}{3} T^{2}+\frac{\theta_{1}}{12} T^{3}\right]\right]\right\}+\left\{\frac { 2 } { 3 } \left[\frac{a_{2}}{T}+C_{2} \lambda_{2}\left[1+\frac{\alpha_{2} T}{2}+\frac{\theta_{2}}{6} T^{2}\right]\right.\right. \\
& +\left(h_{2}+I_{c}\right) C_{2} \lambda_{2}\left[\frac{T}{2}+\frac{\alpha_{2}}{3} T^{2}+\frac{\theta_{2}}{12} T^{3}\right]-\frac{\alpha_{2} r}{2} \\
& -\frac{r C_{2} \lambda_{2}}{2}\left[T+\frac{\alpha_{2}}{2} T^{2}+\frac{\theta_{2}}{6} T^{3}\right]-\left(h_{2}+I_{c}\right) \frac{C_{2} \lambda_{2} r}{2}\left[\frac{T}{2}+\right. \\
& \left.\left.\left.\frac{\alpha_{2}}{3} T^{2}+\frac{\theta_{2}}{12} T^{3}\right]\right]\right\}+\left\{\frac { 1 } { 6 } \left[\frac{a_{3}}{T}+C_{3} \lambda_{3}\left[1+\frac{\alpha_{3} T}{2}+\frac{\theta_{3}}{6} T^{2}\right]\right.\right. \\
& +\left(h_{3}+I_{c}\right) C_{3} \lambda_{3}\left[\frac{T}{2}+\frac{\alpha_{3}}{3} T^{2}+\frac{\theta_{3}}{12} T^{3}\right]-\frac{\alpha_{3} r}{2} \\
& -\frac{r C_{3} \lambda_{3}}{2}\left[T+\frac{\alpha_{3}}{2} T^{2}+\frac{\theta_{3}}{6} T^{3}\right]-\left(h_{3}+I_{c}\right) \frac{C_{3} \lambda_{3} r}{2}\left[\frac{T}{2}\right. \\
& \left.\left.\left.\left.+\frac{\alpha_{3}}{3} T^{2}+\frac{\theta_{3}}{12} T^{3}\right]\right]\right\}\right\}\left(H\left(1+\frac{r H}{2}\right)\right)
\end{aligned}
$$

The necessary condition for minimizing the total cost is $\frac{\partial Z_{1(g m)}(T)}{\partial T}=0$ provided that they satisfy the sufficient conditions $\frac{\partial^{2} Z_{1(g m)}(T)}{\partial T^{2}}>0$

$$
\begin{aligned}
& \left\{\left\{\frac { 1 } { 6 } \left[\frac{-a_{1}}{T^{2}}+C_{1} \lambda_{1}\left[\frac{\alpha_{1}}{2}+\frac{\theta_{1}}{3} T-\frac{r}{2}-\frac{r \alpha_{1}}{2} T+\frac{r \theta_{1}}{4} T^{2}\right]\right.\right.\right. \\
& +\left(h_{1}+I_{c}\right) C_{1} \lambda_{1}\left[\frac{1}{2}+\frac{2 \alpha_{1}}{3} T+\frac{\theta_{1}}{4} T^{2}-\frac{r T}{2}-\frac{r \alpha_{1}}{2} T^{2}\right. \\
& \left.\left.\left.-\frac{r \theta_{1}}{3} T^{3}\right]\right]\right\}+\left\{\frac { 2 } { 3 } \left[\frac{-a_{2}}{T^{2}}+C_{2} \lambda_{2}\left[\frac{\alpha_{2}}{2}+\frac{\theta_{2}}{3} T-\frac{r}{2}-\frac{r \alpha_{2}}{2} T\right.\right.\right. \\
& \left.+\frac{r \theta_{2}}{4} T^{2}\right]+\left(h_{2}+I_{c}\right) C_{2} \lambda_{2}\left[\frac{1}{2}+\frac{2 \alpha_{2}}{3} T+\frac{\theta_{2}}{4} T^{2}-\frac{r T}{2}\right. \\
& \left.\left.\left.-\frac{r \alpha_{2}}{2} T^{2}-\frac{r \theta_{2}}{3} T^{3}\right]\right]\right\}+\left\{\frac { 1 } { 6 } \left[\frac{-a_{3}}{T^{2}}+C_{3} \lambda_{3}\left[\frac{\alpha_{3}}{2}+\frac{\theta_{3}}{3} T-\frac{r}{2}\right.\right.\right. \\
& \left.-\frac{r \alpha_{3}}{2} T+\frac{r \theta_{3}}{4} T^{2}\right]+\left(h_{3}+I_{c}\right) C_{3} \lambda_{3}\left[\frac{1}{2}+\frac{2 \alpha_{3}}{3} T+\frac{\theta_{3}}{4} T^{2}\right. \\
& \left.\left.\left.\left.-\frac{r T}{2}-\frac{r \alpha_{3}}{2} T^{2}-\frac{r \theta_{3}}{3} T^{3}\right]\right]\right\}\right\}\left(H\left(1+\frac{r H}{2}\right)\right)=0
\end{aligned}
$$

Case(ii) $T_{d} \leq m<T$

$$
\begin{aligned}
& \widetilde{Z_{2}(T)}=\left[\frac{A}{T}+C \lambda\left[1+\frac{\alpha T}{2}+\frac{\theta}{6} T^{2}\right]+h C \lambda\left[\frac{T}{2}+\frac{\alpha}{3} T^{2}\right.\right. \\
& \left.+\frac{\theta}{12} T^{3}\right]-\frac{A r}{2}-\frac{r C \lambda}{2}\left(T+\frac{\alpha}{2} T^{2}+\frac{\theta}{6} T^{3}\right)-\frac{h C \lambda r}{2}\left[\frac{T^{2}}{2}\right. \\
& \left.+\frac{\alpha}{3} T^{3}+\frac{\lambda}{12} T^{4}\right]-I_{d} P \lambda\left(m+\frac{T}{2}(m \alpha-1)\right) \\
& \left.+\frac{I_{d} P \lambda r}{2}\left(m T+\frac{T^{2}}{2}(m \alpha-1)\right)\right]\left(H\left(1+\frac{r H}{2}\right)\right)
\end{aligned}
$$

Now defuzzifing the total cost $\widetilde{Z_{2}(T)}$ by using Signed distance method, we have

$$
\begin{aligned}
& Z_{2(s d)}(T)=\frac{1}{4}\left\{Z_{2\left(s d_{1}\right)}(T)+2 Z_{2\left(s d_{2}\right)}^{\widetilde{C}}(T)+Z_{2\left(s d_{3}\right)}(T)\right\} \\
& =\left\{\left\{\frac { 1 } { 4 } \left[\frac{a_{1}}{T}+C_{1} \lambda_{1}\left[1+\frac{\alpha_{1} T}{2}+\frac{\theta_{1}}{6} T^{2}\right]+h_{1} C_{1} \lambda_{1}\left[\frac{T}{2}\right.\right.\right.\right. \\
& \left.+\frac{\alpha_{1}}{3} T^{2}+\frac{\theta_{1}}{12} T^{3}\right]-\frac{\alpha_{1} r}{2}-\frac{r C_{1} \lambda_{1}}{2}\left(T+\frac{\alpha_{1}}{2} T^{2}+\frac{\theta_{1}}{6} T^{3}\right) \\
& -\frac{h_{1} \lambda_{1} r}{2}\left[\frac{T^{2}}{2}+\frac{\alpha_{1}}{3} T^{3}+\frac{\theta_{1}}{12} T^{4}\right]-I_{d} P \lambda_{1}\left(m_{1}\right.
\end{aligned}
$$


$\left.\left.\left.+\frac{T}{2}\left(m_{1} \alpha_{1}-1\right)\right)+\frac{I_{d} P \lambda_{1} r}{2}\left(m_{1} T+\frac{T^{2}}{2}\left(m_{1} \alpha_{1}-1\right)\right)\right]\right\}$

$+\left\{\frac{1}{2}\left[\frac{a_{2}}{T}+C_{2} \lambda_{2}\left[1+\frac{\alpha_{2} T}{2}+\frac{\theta_{2}}{6} T^{2}\right]+h_{2} C_{2} \lambda_{2}\left[\frac{T}{2}+\frac{\alpha_{2}}{3} T^{2}\right.\right.\right.$

$\left.+\frac{\theta_{2}}{12} T^{3}\right]-\frac{\alpha_{2} r}{2}-\frac{r C_{2} \lambda_{2}}{2}\left(T+\frac{\alpha_{2}}{2} T^{2}+\frac{\theta_{2}}{6} T^{3}\right)$

$-\frac{h_{2} C_{2} \lambda_{2} r}{2}\left[\frac{T^{2}}{2}+\frac{\alpha_{2}}{3} T^{3}+\frac{\theta_{2}}{12} T^{4}\right]-I_{d} P \lambda_{2}\left(m_{2}\right.$

$\left.\left.\left.+\frac{T}{2}\left(m_{2} \alpha_{2}-1\right)\right)+\frac{I_{d} P \lambda_{2} r}{2}\left(m_{2} T+\frac{T^{2}}{2}\left(m_{2} \alpha_{2}-1\right)\right)\right]\right\}$

$+\left\{\frac{1}{4}\left[\frac{a_{3}}{T}+C_{3} \lambda_{3}\left[1+\frac{\alpha_{3} T}{2}+\frac{\theta_{3}}{6} T^{2}\right]+h_{3} C_{3} \lambda_{3}\left[\frac{T}{2}+\frac{a_{3}}{3} T^{2}\right.\right.\right.$

$\left.+\frac{\theta_{3}}{12} T^{3}\right]-\frac{\alpha_{3} r}{2}-\frac{r C_{3} \lambda_{3}}{2}\left(T+\frac{\alpha_{3}}{2} T^{2}+\frac{\theta_{3}}{6} T^{3}\right)$

$-\frac{h_{3} \lambda_{3} r}{2}\left[\frac{T^{2}}{2}+\frac{\alpha_{3}}{3} T^{3}+\frac{\theta_{3}}{12} T^{4}\right]-I_{d} P \lambda_{3}\left(m_{3}+\frac{T}{2}\left(m_{3} \alpha_{3}\right.\right.$

$\left.\left.-1))+\frac{I_{d} P \lambda_{3} r}{2}\left(m_{3} T+\frac{T^{2}}{2}\left(m_{3} \alpha_{3}-1\right)\right)\right]\right\}\left(H\left(1+\frac{r H}{2}\right)\right)$

The necessary condition for minimizing the total cost is $\frac{\partial Z_{4(g m)}(T)}{\partial T}=0$ provided that they satisfy the sufficient conditions $\frac{\partial^{2} Z_{4(g m)}(T)}{\partial T^{2}}>0$

$\left\{\left\{\frac{1}{4}\left[\frac{-a_{1}}{T^{2}}+C_{1} \lambda_{1}\left[\frac{\alpha_{1}}{2}+\frac{\theta_{1}}{3} T-\frac{r}{2}-\frac{r \alpha_{1}}{2} T+\frac{r \theta_{1}}{4} T^{2}\right]\right.\right.\right.$

$+h_{1} C_{1} \lambda_{1}\left[\frac{1}{2}+\frac{2 \alpha_{1}}{3} T+\frac{\theta_{1}}{4} T^{2}-\frac{r T}{2}-\frac{r \alpha_{1}}{2} T^{2}-\frac{r \theta_{1}}{6} T^{3}\right]$

$\left.\left.-\frac{I_{d} P \lambda_{1}}{2}\left[\left(m_{1} \alpha_{1}-1\right)(1-r T)-m_{1} r\right]\right]\right\}+\left\{\frac{1}{2}\left[\frac{-a_{2}}{T^{2}}\right.\right.$

$+C_{2} \lambda_{2}\left[\frac{\alpha_{2}}{2}+\frac{\theta_{2}}{3} T-\frac{r}{2}-\frac{r \alpha_{2}}{2} T+\frac{r \theta_{2}}{4} T^{2}\right]+h_{2} C_{2} \lambda_{2}\left[\frac{1}{2}\right.$

$\left.+\frac{2 \alpha_{2}}{3} T+\frac{\theta_{2}}{4} T^{2}-\frac{r T}{2}-\frac{r \alpha_{2}}{2} T^{2}-\frac{r \theta_{2}}{3} T^{3}\right]$

$\left.\left.-\frac{I_{d} P \lambda_{2}}{2}\left[\left(m_{2} \alpha_{2}-1\right)(1-r T)-m_{2} r\right]\right]\right\}+\left\{\frac{1}{4}\left[\frac{-a_{3}}{T^{2}}\right.\right.$

$+C_{3} \lambda_{3}\left[\frac{\alpha_{3}}{2}+\frac{\theta_{3}}{3} T-\frac{r}{2}-\frac{r \alpha_{3}}{2} T+\frac{r \theta_{3}}{4} T^{2}\right]+h_{3} C_{3} \lambda_{3}\left[\frac{1}{2}\right.$

$\left.+\frac{2 \alpha_{3}}{3} T+\frac{\theta_{3}}{4} T^{2}-\frac{r T}{2}-\frac{r \alpha_{3}}{2} T^{2}-\frac{r \alpha_{3}}{3} T^{3}\right]$

$\left.\left.\left.-\frac{I_{d} P \lambda_{3}}{2}\left[\left(m_{3} \alpha_{3}-1\right)(1-r T)-m_{3} r\right]\right]\right\}\right\}\left(H\left(1+\frac{r H}{2}\right)\right)=0$

Now defuzzifing the total cost $\widetilde{Z_{2}(T)}$ by using Graded mean integration method, we have

$$
\begin{aligned}
& Z_{2(g m)}(T)=\frac{1}{6}\left\{Z_{2\left(g m_{1}\right)}(T)+4 Z_{2\left(g m_{2}\right)}^{\sim}(T)+Z_{2\left(g m_{3}\right)}(T)\right\} \\
& =\left\{\left\{\frac { 1 } { 6 } \left[\frac{a_{1}}{T}+C_{1} \lambda_{1}\left[1+\frac{\alpha_{1} T}{2}+\frac{\theta_{1}}{6} T^{2}\right]+h_{1} C_{1} \lambda_{1}\left[\frac{T}{2}\right.\right.\right.\right. \\
& \left.+\frac{\alpha_{1}}{3} T^{2}+\frac{\theta_{1}}{12} T^{3}\right]-\frac{\alpha_{1} r}{2}-\frac{r C_{1} \lambda_{1}}{2}\left(T+\frac{\alpha_{1}}{2} T^{2}+\frac{\theta_{1}}{6} T^{3}\right) \\
& -\frac{h_{1} \lambda_{1} r}{2}\left[\frac{T^{2}}{2}+\frac{\alpha_{1}}{3} T^{3}+\frac{\theta_{1}}{12} T^{4}\right]-I_{d} P \lambda_{1}\left(m_{1}\right. \\
& \left.\left.\left.+\frac{T}{2}\left(m_{1} \alpha_{1}-1\right)\right)+\frac{I_{d} P \lambda_{1} r}{2}\left(m_{1} T+\frac{T^{2}}{2}\left(m_{1} \alpha_{1}-1\right)\right)\right]\right\}
\end{aligned}
$$

$$
\begin{aligned}
& +\left\{\frac { 2 } { 3 } \left[\frac{a_{2}}{T}+C_{2} \lambda_{2}\left[1+\frac{\alpha_{2} T}{2}+\frac{\theta_{2}}{6} T^{2}\right]\right.\right. \\
& +h_{2} C_{2} \lambda_{2}\left[\frac{T}{2}+\frac{\alpha_{2}}{3} T^{2}+\frac{\theta_{2}}{12} T^{3}\right]-\frac{\alpha_{2} r}{2}-\frac{r C_{2} \lambda_{2}}{2}(T+ \\
& \left.\frac{\alpha_{2}}{2} T^{2}+\frac{\theta_{2}}{6} T^{3}\right)-\frac{h_{2} C_{2} \lambda_{2} r}{2}\left[\frac{T^{2}}{2}+\frac{\alpha_{2}}{3} T^{3}+\frac{\theta_{2}}{12} T^{4}\right] \\
& -I_{d} P \lambda_{2}\left(m_{2}+\frac{T}{2}\left(m_{2} \alpha_{2}-1\right)\right)+\frac{I_{d} P \lambda_{2} r}{2}\left(m_{2} T\right. \\
& \left.\left.\left.+\frac{T^{2}}{2}\left(m_{2} \alpha_{2}-1\right)\right)\right]\right\}+\left\{\frac { 1 } { 6 } \left[\frac{a_{3}}{T}+C_{3} \lambda_{3}\left[1+\frac{\alpha_{3} T}{2}+\frac{\theta_{3}}{6} T^{2}\right]\right.\right. \\
& +h_{3} C_{3} \lambda_{3}\left[\frac{T}{2}+\frac{\alpha_{3}}{3} T^{2}+\frac{\theta_{3}}{12} T^{3}\right]-\frac{\alpha_{3} r}{2}-\frac{r C_{3} \lambda_{3}}{2}(T \\
& \left.+\frac{\alpha_{3}}{2} T^{2}+\frac{\theta_{3}}{6} T^{3}\right)-\frac{h_{3} \lambda_{3} r}{2}\left[\frac{T^{2}}{2}+\frac{\alpha_{3}}{3} T^{3}+\frac{\theta_{3}}{12} T^{4}\right] \\
& -I_{d} P \lambda_{3}\left(m_{3}+\frac{T}{2}\left(m_{3} \alpha_{3}-1\right)\right) \\
& \left.\left.+\frac{I_{d} P \lambda_{3} r}{2}\left(m_{3} T+\frac{T^{2}}{2}\left(m_{3} \alpha_{3}-1\right)\right)\right]\right\}\left(H\left(1+\frac{r H}{2}\right)\right)
\end{aligned}
$$

The necessary condition for minimizing the total cost is $\frac{\partial Z_{2(g m)}(T)}{\partial T}=0$ provided that they satisfy the sufficient conditions $\frac{\partial^{2} Z_{2(g m)}(T)}{\partial T^{2}}>0$

$$
\begin{aligned}
& \left\{\left\{\frac { 1 } { 6 } \left[\frac{-a_{1}}{T^{2}}+C_{1} \lambda_{1}\left[\frac{\alpha_{1}}{2}+\frac{\theta_{1}}{3} T-\frac{r}{2}-\frac{r \alpha_{1}}{2} T-\frac{r \theta_{1}}{4} T^{2}\right]\right.\right.\right. \\
& +h_{1} C_{1} \lambda_{1}\left[\frac{1}{2}+\frac{2 \alpha_{1}}{3} T+\frac{\theta_{1}}{4} T^{2}-\frac{r T}{2}-\frac{r \alpha_{1}}{2} T^{2}-\frac{r \theta_{1}}{6} T^{3}\right] \\
& \left.\left.-\frac{I_{d} P \lambda_{1}}{2}\left[\left(m_{1} \alpha_{1}-1\right)(1-r T)-m_{1} r\right]\right]\right\}+\left\{\frac { 2 } { 3 } \left[\frac{-a_{2}}{T^{2}}\right.\right. \\
& +C_{2} \lambda_{2}\left[\frac{\alpha_{2}}{2}+\frac{\theta_{2}}{3} T-\frac{r}{2}-\frac{r \alpha_{2}}{2} T-\frac{r \theta_{2}}{4} T^{2}\right] \\
& +h_{2} C_{2} \lambda_{2}\left[\frac{1}{2}+\frac{2 \alpha_{2}}{3} T+\frac{\theta_{2}}{4} T^{2}-\frac{r T}{2}-\frac{r \alpha_{2}}{2} T^{2}-\frac{r \theta_{2}}{3} T^{3}\right] \\
& \left.\left.-\frac{I_{d} P \lambda_{2}}{2}\left[\left(m_{2} \alpha_{2}-1\right)(1-r T)-m_{2} r\right]\right]\right\}+\left\{\frac { 1 } { 6 } \left[\frac{-a_{3}}{T^{2}}\right.\right. \\
& +C_{3} \lambda_{3}\left[\frac{\alpha_{3}}{2}+\frac{\theta_{3}}{3} T-\frac{r}{2}-\frac{r \alpha_{3}}{2} T-\frac{r \theta_{3}}{4} T^{2}\right]+h_{3} C_{3} \lambda_{3}\left[\frac{1}{2}\right. \\
& \left.+\frac{2 \alpha_{3}}{3} T+\frac{\theta_{3}}{4} T^{2}-\frac{r T}{2}-\frac{r \alpha_{3}}{2} T^{2}-\frac{r \theta_{3}}{3} T^{3}\right] \\
& \left.\left.\left.-\frac{I_{d} P \lambda_{3}}{2}\left[\left(m_{3} \alpha_{3}-1\right)(1-r T)-m_{3} r\right]\right]\right\}\right\}\left(H\left(1+\frac{r H}{2}\right)\right)=0
\end{aligned}
$$

Case(iii) $T_{d} \leq m \leq T$

$\widetilde{Z_{3}(T)}=\left[\frac{A}{T}+C \lambda\left[1+\frac{\alpha T}{2}+\frac{\theta}{6} T^{2}+\frac{h T}{2}+\frac{h a}{3} T^{2}+\frac{h \theta}{12} T^{3}\right.\right.$

$\left.-\frac{r T}{2}-\frac{r \alpha}{4} T^{2}-\frac{r \theta}{12} T^{3}-\frac{h r T^{2}}{4}-\frac{h r \alpha}{6} T^{3}-\frac{h r \theta}{24} T^{4}\right]$

$+I_{c} C \lambda \frac{T}{2}+\frac{\alpha}{3} T^{2}+\frac{\theta}{12} T^{3}-m+\frac{m^{2}}{2 T}-\frac{\alpha m T}{2}+\frac{\alpha m^{3}}{6 T}$

$-\frac{m \theta}{6} T^{2}+\frac{\theta m^{3}}{6}+\frac{\theta m^{4}}{12 T}-\frac{r}{4} T^{2}-\frac{r a}{6} T^{3}-\frac{r \theta}{24} T^{4}+\frac{r m T}{2}$

$\left.-\frac{r m^{2}}{4}+\frac{r m \alpha T^{2}}{4}-\frac{r \alpha m^{3}}{12}+\frac{r m \theta}{12} T^{3}-\frac{r \theta m^{3}}{12} T-\frac{r \theta m^{4}}{24}\right]$

$-\frac{A r}{2}-\frac{I_{d} P \lambda}{2 T}\left(m^{2}(m \alpha+1)\right)$ 


$$
\left.+\frac{I_{d} P \lambda r}{2 \alpha}\left(m^{2}(m \alpha+1)\right)\right]\left(H\left(1+\frac{r H}{2}\right)\right)
$$

Now defuzzifing the total cost $\widetilde{Z_{3}(T)}$ by using Signed distance method, we have

$$
\begin{aligned}
& Z_{3(s d)}(T)=\frac{1}{4}\left\{Z_{3\left(s d_{1}\right)}(T)+2 Z_{3\left(s d_{2}\right)}^{\widetilde{\sim}}(T)+Z_{3\left(s d_{3}\right)}(T)\right\} \\
& =\left\{\frac { 1 } { 4 } \left[\frac{a_{1}}{T}+C_{1} \lambda_{1}\left[1+\frac{\alpha_{1} T}{2}+\frac{\theta_{1}}{6} T^{2}+\frac{h_{1} T}{2}+\frac{h_{1} \alpha_{1}}{3} T^{2}\right.\right.\right. \\
& +\frac{h_{1}}{12} T^{3}-\frac{r T}{2}-\frac{r \alpha_{1}}{4} T^{2}-\frac{r \theta_{1}}{12} T^{3}-\frac{h_{1} r T^{2}}{4}-\frac{h_{1} r \alpha_{1}}{6} T^{3} \\
& \left.-\frac{h_{1} r \theta_{1}}{24} T^{4}\right]+I_{c} C_{1} \lambda_{1}\left[\frac{T}{2}+\frac{\alpha_{1}}{3} T^{2}+\frac{\theta_{1}}{12} T^{3}-m_{1}+\frac{m_{1}^{2}}{2 T}\right. \\
& -\frac{\alpha_{1} m_{1} T}{2}+\frac{\alpha_{1} m_{1}{ }^{3}}{6 T}-\frac{\theta_{1}}{6} T^{2}+\frac{\theta_{1} m_{1}{ }^{3}}{6}+\frac{\theta_{1} m_{1}{ }^{4}}{12 T}-\frac{r}{4} T^{2} \\
& -\frac{r \alpha_{1}}{6} T^{3}-\frac{r \theta_{1}}{24} T^{4}+\frac{r m_{1} T}{2}-\frac{r m_{1}^{2}}{4}+\frac{r m_{1}}{4}-\frac{r \alpha_{1} m_{1}^{3}}{12} \\
& \left.+\frac{r m_{1} \theta_{1}}{12} T^{3}-\frac{r \theta_{1} m_{1}^{3}}{12} T-\frac{r \theta_{1} m_{1}^{4}}{24}\right]-\frac{\alpha_{1} r}{2} \\
& \left.\left.-\frac{I_{d} P \lambda_{1}}{2 T}\left(m_{1}{ }^{2}\left(m_{1} \alpha_{1}+1\right)\right)+\frac{I_{d} P \lambda_{1} r}{2 a_{1}}\left(m_{1}^{2}\left(m_{1}+1\right)\right)\right]\right\} \\
& +\left\{\frac { 1 } { 2 } \left[\frac{a_{2}}{T}+C_{2} \lambda_{2}\left[1+\frac{\alpha_{2} T}{2}+\frac{\theta_{2}}{6} T^{2}+\frac{h_{2} T}{2}+\frac{h_{2} \alpha_{2}}{3} T^{2}\right.\right.\right. \\
& +\frac{h_{2}}{12} T^{3}-\frac{r T}{2}-\frac{r \alpha_{2}}{4} T^{2}-\frac{r \theta_{2}}{12} T^{3}-\frac{h_{2} r T^{2}}{4}-\frac{h_{2} r \alpha_{2}}{6} T^{3} \\
& \left.-\frac{h_{2} r \theta_{2}}{24} T^{4}\right]+I_{c} C_{2} \lambda_{2}\left[\frac{T}{2}+\frac{\alpha_{2}}{3} T^{2}+\frac{\theta_{2}}{12} T^{3}-m_{2}+\frac{m_{2}^{2}}{2 T}\right. \\
& -\frac{\alpha_{2} m_{2} T}{2}+\frac{\alpha_{2} m_{2}{ }^{3}}{6 T}-\frac{\theta_{2}}{6} T^{2}+\frac{\theta_{2} m_{2}{ }^{3}}{6}+\frac{\theta_{2} m_{2}{ }^{4}}{12 T}-\frac{r}{4} T^{2} \\
& -\frac{r \alpha_{2}}{6} T^{3}-\frac{r \theta_{2}}{24} T^{4}+\frac{r m_{2} T}{2}-\frac{r m_{2}^{2}}{4}+\frac{r m_{2}}{4}-\frac{r \alpha_{2} m_{2}{ }^{3}}{12} \\
& \left.+\frac{r m_{2} \theta_{2}}{12} T^{3}-\frac{r \theta_{2} m_{2}{ }^{3}}{12} T-\frac{r \theta_{2} m_{2}{ }^{4}}{24}\right]-\frac{\alpha_{2} r}{2} \\
& \left.\left.-\frac{I_{d} P \lambda_{2}}{2 T}\left(m_{2}^{2}\left(m_{2} \alpha_{2}+1\right)\right)+\frac{I_{d} P \lambda_{2} r}{2 \alpha_{2}}\left(m_{2}^{2}\left(m_{2}+1\right)\right)\right]\right\} \\
& +\left\{\frac { 1 } { 4 } \left[\frac{a_{3}}{T}+C_{3} \lambda_{3}\left[1+\frac{\alpha_{3} T}{2}+\frac{\theta_{3}}{6} T^{2}+\frac{h_{3} T}{2}+\frac{h_{3} a_{3}}{3} T^{2}\right.\right.\right. \\
& +\frac{h_{3}}{12} T^{3}-\frac{r T}{2}-\frac{r \alpha_{3}}{4} T^{2}-\frac{r \theta_{3}}{12} T^{3}-\frac{h_{3} r T^{2}}{4}-\frac{h_{3} r \alpha_{3}}{6} T^{3} \\
& \left.-\frac{h_{3} r \theta_{3}}{24} T^{4}\right]+I_{c} C_{3} \lambda_{3}\left[\frac{T}{2}+\frac{a_{3}}{3} T^{2}+\frac{\theta_{3}}{12} T^{3}-m_{3}+\frac{m_{3}{ }^{2}}{2 T}\right. \\
& -\frac{\alpha_{3} m_{3} T}{2}+\frac{\alpha_{3} m_{3}{ }^{3}}{6 T}-\frac{\theta_{3}}{6} T^{2}+\frac{\theta_{3} m_{3}{ }^{3}}{6}+\frac{\theta_{3} m_{3}{ }^{4}}{12 T}-\frac{r}{4} T^{2} \\
& -\frac{r \alpha_{3}}{6} T^{3}-\frac{r \theta_{3}}{24} T^{4}+\frac{r m_{3} T}{2}-\frac{r m_{3}{ }^{2}}{4}+\frac{r m_{3}}{4}-\frac{r \alpha_{3} m_{3}{ }^{3}}{12} \\
& \left.+\frac{r m_{3} \theta_{3}}{12} T^{3}-\frac{r \theta_{3} m_{3}{ }^{3}}{12} T-\frac{r \theta_{3} m_{3}{ }^{4}}{24}\right]-\frac{\alpha_{3} r}{2} \\
& -\frac{I_{d} P \lambda_{3}}{2 T}\left(m_{3}^{2}\left(m_{3} \alpha_{3}+1\right)\right) \\
& \left.\left.+\frac{I_{d} P \lambda_{3} r}{2 \alpha_{3}}\left(m_{3}{ }^{2}\left(m_{3}+1\right)\right)\right]\right\}\left(H\left(1+\frac{r H}{2}\right)\right)
\end{aligned}
$$

$\left\{\left\{\frac{1}{4}\left[\frac{-a_{1}}{T^{2}}+C_{1} \lambda_{1}\left[\frac{\alpha_{1}}{2}+\frac{\theta_{1}}{3} T-\frac{r}{2}-\frac{r \alpha_{1}}{2} T-\frac{r \theta_{1}}{4} T^{2}\right]\right.\right.\right.$ $+h_{1} C_{1} \lambda_{1}\left[\frac{1}{2}+\frac{2 \alpha_{1}}{3} T+\frac{\theta_{1}}{4} T^{2}-\frac{r T}{2}-\frac{r \alpha_{1}}{2} T^{2}-\frac{r \theta_{1}}{6} T^{3}\right]$

$$
\begin{aligned}
& +C_{1} \lambda_{1} I_{c}\left[\frac{1}{2}+\frac{2 \alpha_{1}}{3} T+\frac{\theta_{1}}{4} T^{2}-\frac{m_{1}^{2}}{2 T^{2}}-\frac{\alpha_{1} m_{1}}{2}-\frac{\alpha_{1} m_{1}^{3}}{6 T^{2}}\right. \\
& -\frac{\theta_{1} m_{1} T}{3}-\frac{\theta_{1} m_{1}^{4}}{12 T^{2}}-\frac{r T}{2}-\frac{r \alpha_{1}}{2} T^{2}-\frac{r \theta_{1}}{6} T^{3}+\frac{m r}{2} \\
& \left.+\frac{m_{1} r \alpha_{1}}{2} T+\frac{m_{1} r \theta_{1}}{4} T^{2}+\frac{r \theta_{1} m_{1}^{3}}{12}\right]+\frac{I_{d} P \lambda_{1}}{2 T^{2}}\left(m _ { 1 } { } ^ { 2 } \left(m_{1} \alpha_{1}\right.\right. \\
& +1))]\}+\left\{\frac { 2 } { 3 } \left[\frac{-a_{2}}{T^{2}}+C_{2} \lambda_{2}\left[\frac{\alpha_{2}}{2}+\frac{\theta_{2}}{3} T-\frac{r}{2}-\frac{r \alpha_{2}}{2} T\right.\right.\right. \\
& \left.-\frac{r \theta_{2}}{4} T^{2}\right]+h_{2} C_{2} \lambda_{2}\left[\frac{1}{2}+\frac{2 \alpha_{2}}{3} T+\frac{\theta_{2}}{4} T^{2}-\frac{r T}{2}-\frac{r \alpha_{2}}{2} T^{2}\right. \\
& \left.-\frac{r \theta_{2}}{6} T^{3}\right]+C_{2} \lambda_{2} I_{c}\left[\frac{1}{2}+\frac{2 \alpha_{2}}{3} T+\frac{\theta_{2}}{4} T^{2}-\frac{m_{2}^{2}}{2 T^{2}}-\frac{\alpha_{2} m_{2}}{2}\right. \\
& -\frac{\alpha_{2} m_{2}{ }^{3}}{6 T^{2}}-\frac{\theta_{2} m_{2} T}{3}-\frac{\theta_{2} m_{2}{ }^{4}}{12 T^{2}}-\frac{r T}{2}-\frac{r \alpha_{2}}{2} T^{2}-\frac{r \theta_{2}}{6} T^{3} \\
& \left.+\frac{m r}{2}+\frac{m_{2} r \alpha_{2}}{2} T+\frac{m_{2} r \theta_{2}}{4} T^{2}+\frac{r \theta_{2} m_{2}^{3}}{12}\right] \\
& \left.\left.+\frac{I_{d} P \lambda_{2}}{2 T^{2}}\left(m_{1}^{2}\left(m_{1} \alpha_{2}+1\right)\right)\right]\right\}+\left\{\frac { 1 } { 4 } \left[\frac{-a_{3}}{T^{2}}+C_{3} \lambda_{3}\left[\frac{\alpha_{3}}{2}\right.\right.\right. \\
& \left.+\frac{\theta_{3}}{3} T-\frac{r}{2}-\frac{r \alpha_{3}}{2} T-\frac{r \theta_{3}}{4} T^{2}\right]+h_{3} C_{3} \lambda_{3}\left[\frac{1}{2}+\frac{2 \alpha_{3}}{3} T\right. \\
& \left.+\frac{\theta_{3}}{4} T^{2}-\frac{r T}{2}-\frac{r \alpha_{3}}{2} T^{2}-\frac{r \theta_{3}}{6} T^{3}\right]+C_{3} \lambda_{3} I_{c}\left[\frac{1}{2}+\frac{2 \alpha_{3}}{3} T\right. \\
& +\frac{\theta_{3}}{4} T^{2}-\frac{m_{3}{ }^{2}}{2 T^{2}}-\frac{\alpha_{3} m_{3}}{2}-\frac{\alpha_{3} m_{3}{ }^{3}}{6 T^{2}}-\frac{\theta_{3} m_{3} T}{3}-\frac{\theta_{3} m_{3}{ }^{4}}{12 T^{2}} \\
& -\frac{r T}{2}-\frac{r \alpha_{3}}{2} T^{2}-\frac{r \theta_{3}}{6} T^{3}+\frac{m r}{2}+\frac{m_{3} r \alpha_{3}}{2} T+\frac{m_{3} r \theta_{3}}{4} T^{2} \\
& \left.\left.\left.\left.+\frac{r \theta_{3} m_{3}^{3}}{12}\right]+\frac{I_{d} P \lambda_{3}}{2 T^{2}}\left(m_{3}^{2}\left(m_{3} \alpha_{3}+1\right)\right)\right]\right\}\right\}\left(H\left(1+\frac{r H}{2}\right)\right)=0
\end{aligned}
$$

Now defuzzifing the total cost $\widetilde{Z_{2}(T)}$ by using Graded mean integration method, we have

$$
\begin{aligned}
& Z_{3(g m)}(T)=\frac{1}{6}\left\{Z_{3\left(g m_{1}\right)}(T)+4 Z_{3\left(g m_{2}\right)}(T)+Z_{3\left(g m_{3}\right)}(T)\right\} \\
& =\left\{\frac { 1 } { 6 } \left[\frac{a_{1}}{T}+C_{1} \lambda_{1}\left[1+\frac{\alpha_{1} T}{2}+\frac{\theta_{1}}{6} T^{2}+\frac{h_{1} T}{2}+\frac{h_{1} \alpha_{1}}{3} T^{2}\right.\right.\right. \\
& +\frac{h_{1}}{12} T^{3}-\frac{r T}{2}-\frac{r \alpha_{1}}{4} T^{2}-\frac{r \theta_{1}}{12} T^{3}-\frac{h_{1} r T^{2}}{4}-\frac{h_{1} r \alpha_{1}}{6} T^{3} \\
& \left.-\frac{h_{1} r \theta_{1}}{24} T^{4}\right]+I_{c} C_{1} \lambda_{1}\left[\frac{T}{2}+\frac{\alpha_{1}}{3} T^{2}+\frac{\theta_{1}}{12} T^{3}-m_{1}+\frac{m_{1}{ }^{2}}{2 T}\right. \\
& -\frac{\alpha_{1} m_{1} T}{2}+\frac{\alpha_{1} m_{1}{ }^{3}}{6 T}-\frac{\theta_{1}}{6} T^{2}+\frac{\theta_{1} m_{1}^{3}}{6}+\frac{\theta_{1} m_{1} 4}{12 T}-\frac{r}{4} T^{2} \\
& -\frac{r \alpha_{1}}{6} T^{3}-\frac{r \theta_{1}}{24} T^{4}+\frac{r m_{1} T}{2}-\frac{r m_{1}{ }^{2}}{4}+\frac{r m_{1}}{4}-\frac{r \alpha_{1} m_{1}{ }^{3}}{12} \\
& \left.+\frac{r m_{1} \theta_{1}}{12} T^{3}-\frac{r \theta_{1} m_{1}{ }^{3}}{12} T-\frac{r \theta_{1} m_{1}{ }^{4}}{24}\right]-\frac{\alpha_{1} r}{2} \\
& \left.\left.-\frac{I_{d} P \lambda_{1}}{2 T}\left(m_{1}{ }^{2}\left(m_{1} \alpha_{1}+1\right)\right)+\frac{I_{d} P \lambda_{1} r}{2 \alpha_{1}}\left(m_{1}{ }^{2}\left(m_{1}+1\right)\right)\right]\right\} \\
& +\left\{\frac { 2 } { 3 } \left[\frac{a_{2}}{T}+C_{2} \lambda_{2}\left[1+\frac{\alpha_{2} T}{2}+\frac{\theta_{2}}{6} T^{2}+\frac{h_{2} T}{2}+\frac{h_{2} \alpha_{2}}{3} T^{2}\right.\right.\right. \\
& +\frac{h_{2}}{12} T^{3}-\frac{r T}{2}-\frac{r \alpha_{2}}{4} T^{2}-\frac{r \theta_{2}}{12} T^{3}-\frac{h_{2} r T^{2}}{4}-\frac{h_{2} r \alpha_{2}}{6} T^{3} \\
& \left.-\frac{h_{2} r \theta_{2}}{24} T^{4}\right]+I_{c} C_{2} \lambda_{2}\left[\frac{T}{2}+\frac{\alpha_{2}}{3} T^{2} \frac{\theta_{2}}{12} T^{3}-m_{2}+\frac{m_{2}{ }^{2}}{2 T}\right.
\end{aligned}
$$


$-\frac{\alpha_{2} m_{2} T}{2}+\frac{\alpha_{2} m_{2}^{3}}{6 T}-\frac{\theta_{2}}{6} T^{2}+\frac{\theta_{2} m_{2}^{3}}{6}+\frac{\theta_{2} m_{2}^{4}}{12 T}-\frac{r}{4} T^{2}$

$-\frac{r \alpha_{2}}{6} T^{3}-\frac{r \theta_{2}}{24} T^{4}+\frac{r m_{2} T}{2}-\frac{r m_{2}^{2}}{4}+\frac{r m_{2}}{4}-\frac{r \alpha_{2} m_{2}{ }^{3}}{12}$

$\left.+\frac{r m_{2} \theta_{2}}{12} T^{3}-\frac{r \theta_{2} m_{2}^{3}}{12} T-\frac{r \theta_{2} m_{2}^{4}}{24}\right]-\frac{\alpha_{2} r}{2}$

$\left.\left.-\frac{I_{d} P \lambda_{2}}{2 T}\left(m_{2}^{2}\left(m_{2} \alpha_{2}+1\right)\right)+\frac{I_{d} P \lambda_{2} r}{2 \alpha_{2}}\left(m_{2}^{2}\left(m_{2}+1\right)\right)\right]\right\}$

$+\left\{\frac{1}{6}\left[\frac{a_{3}}{T}+C_{3} \lambda_{3}\left[1+\frac{\alpha_{3} T}{2}+\frac{\theta_{3}}{6} T^{2}+\frac{h_{3} T}{2}+\frac{h_{3} \alpha_{3}}{3} T^{2}\right.\right.\right.$

$+\frac{h_{3}}{12} T^{3}-\frac{r T}{2}-\frac{r \alpha_{3}}{4} T^{2}-\frac{r \theta_{3}}{12} T^{3}-\frac{h_{3} r T^{2}}{4}-\frac{h_{3} r \alpha_{3}}{6} T^{3}$

$\left.-\frac{h_{3} r \theta_{3}}{24} T^{4}\right]+I_{c} C_{3} \lambda_{3}\left[\frac{T}{2}+\frac{\alpha_{3}}{3} T^{2}+\frac{\theta_{3}}{12} T^{3}-m_{3}+\frac{m_{3}^{2}}{2 T}\right.$

$-\frac{\alpha_{3} m_{3} T}{2}+\frac{\alpha_{3} m_{3}{ }^{3}}{6 T}-\frac{\theta_{3}}{6} T^{2}+\frac{\theta_{3} m_{3}{ }^{3}}{6}+\frac{\theta_{3} m_{3}{ }^{4}}{12 T}-\frac{r}{4} T^{2}$

$-\frac{r \alpha_{3}}{6} T^{3}-\frac{r \theta_{3}}{24} T^{4}+\frac{r m_{3} T}{2}-\frac{r m_{3}{ }^{2}}{4}+\frac{r m_{3}}{4}-\frac{r \alpha_{3} m_{3}{ }^{3}}{12}$

$\left.+\frac{r m_{3} \theta_{3}}{12} T^{3}-\frac{r \theta_{3} m_{3}{ }^{3}}{12} T-\frac{r \theta_{3} m_{3}{ }^{4}}{24}\right]-\frac{\alpha_{3} r}{2}$

$-\frac{I_{d} P \lambda_{3}}{2 T}\left(m_{3}^{2}\left(m_{3} \alpha_{3}+1\right)\right)$

$\left.\left.+\frac{I_{d} P \lambda_{3} r}{2 \alpha_{3}}\left(m_{3}{ }^{2}\left(m_{3}+1\right)\right)\right]\right\}\left(H\left(1+\frac{r H}{2}\right)\right)$

The necessary condition for minimizing the total cost is $\frac{\partial Z_{3(g m)}(T)}{\partial T}=0$ provided that they satisfy the sufficient conditions $\frac{\partial^{2} Z_{3(g m)}(T)}{\partial T^{2}}>0$

$\left\{\left\{\frac{1}{4}\left[\frac{-a_{1}}{T^{2}}+C_{1} \lambda_{1}\left[\frac{\alpha_{1}}{2}+\frac{\theta_{1}}{3} T-\frac{r}{2}-\frac{r a_{1}}{2} T-\frac{r \theta_{1}}{4} T^{2}\right]\right.\right.\right.$

$+h_{1} C_{1} \lambda_{1}\left[\frac{1}{2}+\frac{2 \alpha_{1}}{3} T+\frac{\theta_{1}}{4} T^{2}-\frac{r T}{2}-\frac{r \alpha_{1}}{2} T^{2}-\frac{r \theta_{1}}{6} T^{3}\right]$

$+C_{1} \lambda_{1} I_{c}\left[\frac{1}{2}+\frac{2 \alpha_{1}}{3} T+\frac{\theta_{1}}{4} T^{2}-\frac{m_{1}^{2}}{2 T^{2}}-\frac{\alpha_{1} m_{1}}{2}-\frac{\alpha_{1} m_{1}{ }^{3}}{6 T^{2}}\right.$

$-\frac{\theta_{1} m_{1} T}{3}-\frac{\theta_{1} m_{1}^{4}}{12 T^{2}}-\frac{r T}{2}-\frac{r \alpha_{1}}{2} T^{2}-\frac{r \theta_{1}}{6} T^{3}+\frac{m r}{2}$

$\left.+\frac{m_{1} r \alpha_{1}}{2} T+\frac{m_{1} r \theta_{1}}{4} T^{2}+\frac{r \theta_{1} m_{1}^{3}}{12}\right]$

$\left.\left.+\frac{I_{d} P \lambda_{1}}{2 T^{2}}\left(m_{1}^{2}\left(m_{1} \alpha_{1}+1\right)\right)\right]\right\}+\left\{\frac{2}{3}\left[\frac{-a_{2}}{T^{2}}+C_{2} \lambda_{2}\left[\frac{\alpha_{2}}{2}+\frac{\theta_{2}}{3} T\right.\right.\right.$

$\left.-\frac{r}{2}-\frac{r \alpha_{2}}{2} T-\frac{r \theta_{2}}{4} T^{2}\right]+h_{2} C_{2} \lambda_{2}\left[\frac{1}{2}+\frac{2 \alpha_{2}}{3} T+\frac{\theta_{2}}{4} T^{2}-\frac{r T}{2}\right.$

$\left.-\frac{r \alpha_{2}}{2} T^{2}-\frac{r \theta_{2}}{6} T^{3}\right]+C_{2} \lambda_{2} I_{c}\left[\frac{1}{2}+\frac{2 \alpha_{2}}{3} T+\frac{\theta_{2}}{4} T^{2}-\frac{m_{2}{ }^{2}}{2 T^{2}}\right.$

$-\frac{\alpha_{2} m_{2}}{2}-\frac{\alpha_{2} m_{2}{ }^{3}}{6 T^{2}}-\frac{\theta_{2} m_{2} T}{3}-\frac{\theta_{2} m_{2}{ }^{4}}{12 T^{2}}-\frac{r T}{2}-\frac{r \alpha_{2}}{2} T^{2}$

$\left.-\frac{r \theta_{2}}{6} T^{3}+\frac{m r}{2}+\frac{m_{2} r \alpha_{2}}{2} T+\frac{m_{2} r \theta_{2}}{4} T^{2}+\frac{r \theta_{2} m_{2}{ }^{3}}{12}\right]$

$\left.\left.+\frac{I_{d} P \lambda_{2}}{2 T^{2}}\left(m_{2}{ }^{2}\left(m_{2} \alpha_{2}+1\right)\right)\right]\right\}+\left\{\frac{1}{4}\left[\frac{-a_{3}}{T^{2}}+C_{3} \lambda_{3}\left[\frac{\alpha_{3}}{2}\right.\right.\right.$

$\left.+\frac{\theta_{3}}{3} T-\frac{r}{2}-\frac{r \alpha_{3}}{2} T-\frac{r \theta_{3}}{4} T^{2}\right]+h_{3} C_{3} \lambda_{3}\left[\frac{1}{2}+\frac{2 \alpha_{3}}{3} T\right.$

$\left.+\frac{\theta_{3}}{4} T^{2}-\frac{r T}{2}-\frac{r \alpha_{3}}{2} T^{2}-\frac{r \theta_{3}}{6} T^{3}\right]+C_{3} \lambda_{3} I_{c}\left[\frac{1}{2}+\frac{2 \alpha_{3}}{3} T\right.$ $+\frac{\theta_{3}}{4} T^{2}-\frac{m_{3}{ }^{2}}{2 T^{2}}-\frac{\alpha_{3} m_{3}}{2}-\frac{\alpha_{3} m_{3}{ }^{3}}{6 T^{2}}-\frac{\theta_{3} m_{3} T}{3}-\frac{\theta_{3} m_{3}{ }^{4}}{12 T^{2}}$

$-\frac{r T}{2}-\frac{r \alpha_{3}}{2} T^{2}-\frac{r \theta_{3}}{6} T^{3}+\frac{m r}{2}+\frac{m_{3} r \alpha_{3}}{2} T+\frac{m_{3} r \theta_{3}}{4} T^{2}$

$\left.\left.\left.\left.+\frac{r \theta_{3} m_{3}{ }^{3}}{12}\right]+\frac{I_{d} P \lambda_{3}}{2 T^{2}}\left(m_{3}^{2}\left(m_{3} \alpha_{3}+1\right)\right)\right]\right\}\right\}$

$\times\left(H\left(1+\frac{r H}{2}\right)\right)=0$

Case (iv) $m \leq T_{d} \leq T$

$\widetilde{Z_{4}(T)}=\left[\frac{A}{T}+C \lambda\left[1+\frac{\alpha T}{2}+\frac{\theta}{6} T^{2}+\frac{h T}{2}+\frac{h \alpha}{3} T^{2}\right.\right.$

$+\frac{h \theta}{12} T^{3}-\frac{r T}{2}-\frac{r \alpha}{4} T^{2}-\frac{r \theta}{12} T^{3}-\frac{h r T^{2}}{4}-\frac{h r \alpha}{6} T^{3}$

$\left.-\frac{h r \theta}{24} T^{4}\right]+I_{c} C \lambda\left[\frac{T}{2}+\frac{\alpha}{3} T^{2}+\frac{\theta}{12} T^{3}-m+\frac{m^{2}}{2 T}\right.$

$-\frac{\alpha m T}{2}+\frac{\alpha m^{3}}{6 T}-\frac{m \theta}{6} T^{2}+\frac{\theta m^{3}}{6}+\frac{\theta m^{4}}{12 T}-\frac{r}{4} T^{2}-\frac{r \alpha}{6} T^{3}$

$-\frac{r \theta}{24} T^{4}+\frac{r m T}{2}-\frac{r m^{2}}{4}+\frac{r m \alpha T^{2}}{4}-\frac{r \alpha m^{3}}{12}+\frac{r m \theta}{12} T^{3}$

$\left.-\frac{r \theta m^{3}}{12} T-\frac{r \theta m^{4}}{24}\right]-\frac{A r}{2}-\frac{I_{d} P \lambda}{2 T}\left(m^{2}(m \alpha+1)\right)$

$\left.+\frac{I_{d} P \lambda r}{2 \alpha}\left(m^{2}(m \alpha+1)\right)\right]\left(H\left(1+\frac{r H}{2}\right)\right)$

Now defuzzifing the total cost $\widetilde{Z_{4}(T)}$ by using Signed distance method, we have

$$
\begin{aligned}
& Z_{4(s d)}(T)=\frac{1}{4}\left\{Z_{4\left(s d_{1}\right)}(T)+2 \underset{4\left(s d_{2}\right)}{\sim}(T)+Z_{4\left(s d_{3}\right)}(T)\right\} \\
& =\left\{\frac { 1 } { 4 } \left[\frac{a_{1}}{T}+C_{1} \lambda_{1}\left[1+\frac{\alpha_{1} T}{2}+\frac{\theta_{1}}{6} T^{2}+\frac{h_{1} T}{2}+\frac{h_{1} \alpha_{1}}{3} T^{2}\right.\right.\right. \\
& +\frac{h_{1}}{12} T^{3}-\frac{r T}{2}-\frac{r \alpha_{1}}{4} T^{2}-\frac{r \theta_{1}}{12} T^{3}-\frac{h_{1} r T^{2}}{4}-\frac{h_{1} r \alpha_{1}}{6} T^{3} \\
& \left.-\frac{h_{1} r \theta_{1}}{24} T^{4}\right]+I_{c} C_{1} \lambda_{1}\left[\frac{T}{2}+\frac{\alpha_{1}}{3} T^{2}+\frac{\theta_{1}}{12} T^{3}-m_{1}+\frac{m_{1}{ }^{2}}{2 T}\right. \\
& -\frac{\alpha_{1} m_{1} T}{2}+\frac{\alpha_{1} m_{1}^{3}}{6 T}-\frac{\theta_{1}}{6} T^{2}+\frac{\theta_{1} m_{1}{ }^{3}}{6}+\frac{\theta_{1} m_{1}{ }^{4}}{12 T}-\frac{r}{4} T^{2} \\
& -\frac{r \alpha_{1}}{6} T^{3}-\frac{r \theta_{1}}{24} T^{4}+\frac{r m_{1} T}{2}-\frac{r m_{1}{ }^{2}}{4}+\frac{r m_{1}}{4}-\frac{r \alpha_{1} m_{1}{ }^{3}}{12} \\
& \left.+\frac{r m_{1} \theta_{1}}{12} T^{3}-\frac{r \theta_{1} m_{1}{ }^{3}}{12} T-\frac{r \theta_{1} m_{1}{ }^{4}}{24}\right]-\frac{\alpha_{1} r}{2} \\
& \left.\left.-\frac{I_{d} P \lambda_{1}}{2 T}\left(m_{1}{ }^{2}\left(m_{1} \alpha_{1}+1\right)\right)+\frac{I_{d} P \lambda_{1} r}{2 \alpha_{1}}\left(m_{1}{ }^{2}\left(m_{1}+1\right)\right)\right]\right\} \\
& +\left\{\frac { 1 } { 2 } \left[\frac{a_{2}}{T}+C_{2} \lambda_{2}\left[1+\frac{\alpha_{2} T}{2}+\frac{\theta_{2}}{6} T^{2}+\frac{h_{2} T}{2}+\frac{h_{2} \alpha_{2}}{3} T^{2}\right.\right.\right. \\
& +\frac{h_{2}}{12} T^{3}-\frac{r T}{2}-\frac{r \alpha_{2}}{4} T^{2}-\frac{r \theta_{2}}{12} T^{3}-\frac{h_{2} r T^{2}}{4}-\frac{h_{2} r \alpha_{2}}{6} T^{3} \\
& \left.-\frac{h_{2} r \theta_{2}}{24} T^{4}\right]+I_{c} C_{2} \lambda_{2}\left[\frac{T}{2}+\frac{\alpha_{2}}{3} T^{2}+\frac{\theta_{2}}{12} T^{3}-m_{2}+\frac{m_{2}{ }^{2}}{2 T}\right. \\
& -\frac{\alpha_{2} m_{2} T}{2}+\frac{\alpha_{2} m_{2}{ }^{3}}{6 T}-\frac{\theta_{2}}{6} T^{2}+\frac{\theta_{2} m_{2}{ }^{3}}{6}+\frac{\theta_{2} m_{2}{ }^{4}}{12 T}-\frac{r}{4} T^{2} \\
& -\frac{r \alpha_{2}}{6} T^{3}-\frac{r \theta_{2}}{24} T^{4}+\frac{r m_{2} T}{2}-\frac{r m_{2}{ }^{2}}{4}+\frac{r m_{2}}{4}-\frac{r \alpha_{2} m_{2}{ }^{3}}{12} \\
& \left.+\frac{r m_{2} \theta_{2}}{12} T^{3}-\frac{r \theta_{2} m_{2}{ }^{3}}{12} T-\frac{r \theta_{2} m_{2}{ }^{4}}{24}\right]-\frac{\alpha_{2} r}{2}
\end{aligned}
$$




$$
\begin{aligned}
& \left.\left.-\frac{I_{d} P \lambda_{2}}{2 T}\left(m_{2}^{2}\left(m_{2} \alpha_{2}+1\right)\right)+\frac{I_{d} P \lambda_{2} r}{2 \alpha_{2}}\left(m_{2}^{2}\left(m_{2}+1\right)\right)\right]\right\} \\
& +\left\{\frac { 1 } { 4 } \left[\frac{a_{3}}{T}+C_{3} \lambda_{3}\left[1+\frac{\alpha_{3} T}{2}+\frac{\theta_{3}}{6} T^{2}+\frac{h_{3} T}{2}+\frac{h_{3} \alpha_{3}}{3} T^{2}\right.\right.\right. \\
& +\frac{h_{3}}{12} T^{3}-\frac{r T}{2}-\frac{r \alpha_{3}}{4} T^{2}-\frac{r \theta_{3}}{12} T^{3}-\frac{h_{3} r T^{2}}{4}-\frac{h_{3} r \alpha_{3}}{6} T^{3} \\
& \left.-\frac{h_{3} r \theta_{3}}{24} T^{4}\right]+I_{c} C_{3} \lambda_{3}\left[\frac{T}{2}+\frac{\alpha_{3}}{3} T^{2}+\frac{\theta_{3}}{12} T^{3}-m_{3}+\frac{m_{3}{ }^{2}}{2 T}\right. \\
& -\frac{\alpha_{3} m_{3} T}{2}+\frac{\alpha_{3} m_{3}{ }^{3}}{6 T}-\frac{\theta_{3}}{6} T^{2}+\frac{\theta_{3} m_{3}{ }^{3}}{6}+\frac{\theta_{3} m_{3}{ }^{4}}{12 T}-\frac{r}{4} T^{2} \\
& -\frac{r \alpha_{3}}{6} T^{3}-\frac{r \theta_{3}}{24} T^{4}+\frac{r m_{3} T}{2}-\frac{r m_{3}{ }^{2}}{4}+\frac{r m_{3}}{4}-\frac{r \alpha_{3} m_{3}{ }^{3}}{12} \\
& \left.+\frac{r m_{3} \theta_{3}}{12} T^{3}-\frac{r \theta_{3} m_{3}{ }^{3}}{12} T-\frac{r \theta_{3} m_{3}{ }^{4}}{24}\right]-\frac{\alpha_{3} r}{2} \\
& -\frac{I_{d} P \lambda_{3}}{2 T}\left(m_{3}{ }^{2}\left(m_{3} \alpha_{3}+1\right)\right) \\
& \left.\left.+\frac{I_{d} P \lambda_{3} r}{2 \alpha_{3}}\left(m_{3}{ }^{2}\left(m_{3}+1\right)\right)\right]\right\}\left(H\left(1+\frac{r H}{2}\right)\right)
\end{aligned}
$$

The necessary condition for minimizing the total cost is $\frac{\partial Z_{4(s d)}(T)}{\partial T}=0$ provided that they satisfy the sufficient conditions $\frac{\partial^{2} Z_{4(s d)}(T)}{\partial T^{2}}>0$

Now defuzzifing the total cost $\widetilde{Z_{2}(T)}$ by using Graded mean integration method, we have

$$
\begin{aligned}
& Z_{4(g m)}(T)=\frac{1}{6}\left\{Z_{4\left(g m_{1}\right)}(T)+4 Z_{4\left(g m_{2}\right)}(T)+Z_{4\left(g m_{3}\right)}(T)\right\} \\
& =\left\{\frac { 1 } { 6 } \left[\frac{a_{1}}{T}+C_{1} \lambda_{1}\left[1+\frac{\alpha_{1} T}{2}+\frac{\theta_{1}}{6} T^{2}+\frac{h_{1} T}{2}+\frac{h_{1} \alpha_{1}}{3} T^{2}\right.\right.\right. \\
& +\frac{h_{1}}{12} T^{3}-\frac{r T}{2}-\frac{r \alpha_{1}}{4} T^{2}-\frac{r \theta_{1}}{12} T^{3}-\frac{h_{1} r T^{2}}{4}-\frac{h_{1} r \alpha_{1}}{6} T^{3} \\
& \left.-\frac{h_{1} r \theta_{1}}{24} T^{4}\right]+I_{c} C_{1} \lambda_{1}\left[\frac{T}{2}+\frac{\alpha_{1}}{3} T^{2}+\frac{\theta_{1}}{12} T^{3}-m_{1}+\frac{m_{1}^{2}}{2 T}\right. \\
& -\frac{\alpha_{1} m_{1} T}{2}+\frac{\alpha_{1} m_{1}^{3}}{6 T}-\frac{\theta_{1}}{6} T^{2}+\frac{\theta_{1} m_{1}^{3}}{6}+\frac{\theta_{1} m_{1}{ }^{4}}{12 T}-\frac{r}{4} T^{2} \\
& -\frac{r \alpha_{1}}{6} T^{3}-\frac{r \theta_{1}}{24} T^{4}+\frac{r m_{1} T}{2}-\frac{r m_{1}^{2}}{4}+\frac{r m_{1}}{4}-\frac{r \alpha_{1} m_{1}^{3}}{12} \\
& \left.+\frac{r m_{1} \theta_{1}}{12} T^{3}-\frac{r \theta_{1} m_{1}^{3}}{12} T-\frac{r \theta_{1} m_{1}^{4}}{24}\right]-\frac{\alpha_{1} r}{2} \\
& \left.\left.-\frac{I_{d} P \lambda_{1}}{2 T}\left(m_{1}^{2}\left(m_{1} \alpha_{1}+1\right)\right)+\frac{I_{d} P \lambda_{1} r}{2 \alpha_{1}}\left(m_{1}^{2}\left(m_{1}+1\right)\right)\right]\right\} \\
& +\left\{\frac { 2 } { 3 } \left[\frac{a_{2}}{T}+C_{2} \lambda_{2}\left[1+\frac{\alpha_{2} T}{2}+\frac{\theta_{2}}{6} T^{2}+\frac{h_{2} T}{2}+\frac{h_{2} a_{2}}{3} T^{2}\right.\right.\right. \\
& +\frac{h_{2}}{12} T^{3}-\frac{r T}{2}-\frac{r \alpha_{2}}{4} T^{2}-\frac{r \theta_{2}}{12} T^{3}-\frac{h_{2} r T^{2}}{4}-\frac{h_{2} r \alpha_{2}}{6} T^{3} \\
& \left.-\frac{h_{2} r \theta_{2}}{24} T^{4}\right]+I_{c} C_{2} \lambda_{2}\left[\frac{T}{2}+\frac{\alpha_{2}}{3} T^{2}+\frac{\theta_{2}}{12} T^{3}-m_{2}+\frac{m_{2}^{2}}{2 T}\right. \\
& -\frac{\alpha_{2} m_{2} T}{2}+\frac{\alpha_{2} m_{2}{ }^{3}}{6 T}-\frac{\theta_{2}}{6} T^{2}+\frac{\theta_{2} m_{2}{ }^{3}}{6}+\frac{\theta_{2} m_{2}{ }^{4}}{12 T}-\frac{r}{4} T^{2} \\
& -\frac{r \alpha_{2}}{6} T^{3}-\frac{r 2}{24} T^{4}+\frac{r m_{2} T}{2}-\frac{r m_{2}^{2}}{4}+\frac{r m_{2}}{4}-\frac{r \alpha_{2} m_{2}{ }^{3}}{12} \\
& \left.+\frac{r m_{2} \theta_{2}}{12} T^{3}-\frac{r \theta_{2} m_{2}{ }^{3}}{12} T-\frac{r \theta_{2} m_{2}{ }^{4}}{24}\right]-\frac{\alpha_{2} r}{2} \\
& \left.\left.-\frac{I_{d} P \lambda_{2}}{2 T}\left(m_{2}^{2}\left(m_{2} \alpha_{2}+1\right)\right)+\frac{I_{d} P \lambda_{2} r}{2 \alpha_{2}}\left(m_{2}^{2}\left(m_{2}+1\right)\right)\right]\right\}
\end{aligned}
$$

$$
\begin{aligned}
& +\left\{\frac { 1 } { 6 } \left[\frac{a_{3}}{T}+C_{3} \lambda_{3}\left[1+\frac{\alpha_{3} T}{2}+\frac{\theta_{3}}{6} T^{2}+\frac{h_{3} T}{2}+\frac{h_{3} a_{3}}{3} T^{2}\right.\right.\right. \\
& +\frac{h_{3}}{12} T^{3}-\frac{r T}{2}-\frac{r \alpha_{3}}{4} T^{2}-\frac{r \theta_{3}}{12} T^{3}-\frac{h_{3} r T^{2}}{4}-\frac{h_{3} r \alpha_{3}}{6} T^{3} \\
& \left.-\frac{h_{3} r \theta_{3}}{24} T^{4}\right]+I_{c} C_{3} \lambda_{3}\left[\frac{T}{2}+\frac{\alpha_{3}}{3} T^{2}+\frac{\theta_{3}}{12} T^{3}-m_{3}+\frac{m_{3}{ }^{2}}{2 T}\right. \\
& -\frac{\alpha_{3} m_{3} T}{2}+\frac{\alpha_{3} m_{3}{ }^{3}}{6 T}-\frac{\theta_{3}}{6} T^{2}+\frac{\theta_{3} m_{3}{ }^{3}}{6}+\frac{\theta_{3} m_{3} 4}{12 T}-\frac{r}{4} T^{2} \\
& -\frac{r \alpha_{3}}{6} T^{3}-\frac{r 3}{24} T^{4}+\frac{r m_{3} T}{2}-\frac{r m_{3}{ }^{2}}{4}+\frac{r m_{3}}{4}-\frac{r \alpha_{3} m_{3}{ }^{3}}{12} \\
& \left.+\frac{r m_{3} \theta_{3}}{12} T^{3}-\frac{r \theta_{3} m_{3}{ }^{3}}{12} T-\frac{r \theta_{3} m_{3}}{24}\right]-\frac{\alpha_{3} r}{2} \\
& -\frac{I_{d} P \lambda_{3}}{2 T}\left(m_{3}{ }^{2}\left(m_{3} \alpha_{3}+1\right)\right) \\
& \left.\left.+\frac{I_{d} P \lambda_{3} r}{2 \alpha_{3}}\left(m_{3}{ }^{2}\left(m_{3}+1\right)\right)\right]\right\}\left(H\left(1+\frac{r H}{2}\right)\right)=0
\end{aligned}
$$

The necessary condition for minimizing the total cost is $\frac{\partial Z_{4(g m)}(T)}{\partial T}=0$ provided that they satisfy the sufficient conditions $\frac{\partial^{2} Z_{4(g m)}(T)}{\partial T^{2}}>0$

case (iv) is same as case (iii)

\section{Numerical Examples}

Consider an inventory system with the parametric values in proper units

Example : Case(i) $0<T<T_{d}$

Suppose $\tilde{A}=(100,200,300), \tilde{C}=(15,25,35), \tilde{h}=$ $(2,5,8), \tilde{\alpha}=(0.25,0.5,0.75), \tilde{\theta}=(0.1,0.2,0.3), \tilde{\lambda}=$ $(100,200,300), I_{c}=0.10, I_{d}=0.05, r=0.05, H=1$, by signed distance method and graded mean integration we get

$T=0.0902, \widetilde{Z_{1(s d)}}(T)=9715.1$ and $T=0.0870$, $\widetilde{Z_{1(g m)}}(T)=9403.4$

Case(ii) $T_{d} \leq m<T$

Suppose $\overline{\tilde{A}}=(80,160,240), \tilde{C}=(10,20,30), \tilde{h}=$ $(2,5,8), \tilde{\alpha}=(0.2,0.4,0.6), \tilde{\theta}=(0.1,0.2,0.3), \tilde{\lambda}=$ $(50,100,150), \tilde{m}=(5,10,15), r=0.05, n=40, I_{d}=0.01$, $H=1$, by signed distance method and graded mean integration we get

$T=0.1375, \widetilde{Z_{1(s d)}}(T)=4143.2$ and $T=0.1462$, $\widetilde{Z_{1(\mathrm{gm})}}(T)=3951.1$

Case(iii) $T_{d} \leq m \leq T$

Suppose $\tilde{A}=(20,40,60), \tilde{C}=(20,30,40), \tilde{h}=(2,5,8)$, $\tilde{\alpha}=(0.2,0.4,0.6), \tilde{\theta}=(0.1,0.2,0.3), \tilde{\lambda}=(50,75,100)$, $\tilde{m}=(5,10,15), r=0.05, n=40, I_{c}=0.01, I_{d}=0.01$, $H=1$, by signed distance method and graded mean integration we get

$T=0.6803, \widetilde{Z_{1(s d)}}(T)=$ and $T=0.5674, \widetilde{Z_{1(g m)}}(T)=$ 48775

Case (iv) $m \leq T_{d} \leq T$

Case (iv) is same as case(iii).

\section{Sensitivity Analysis}

Case(i) $0<T<T_{d}$ 
Table 1. Effects of different parameters using signed distance method

\begin{tabular}{|c|c|c|c|}
\hline Parameter & & $\mathrm{T}$ & $T C_{G M}(\mathrm{~T})$ \\
\hline \multirow[t]{4}{*}{ A } & $(150,250,350)$ & 0.1005 & 10205 \\
\hline & $(200,300,400)$ & 0.1097 & 10650 \\
\hline & $(250,350,450)$ & 0.1181 & 11061 \\
\hline & $(300,400,500)$ & 0.1258 & 11445 \\
\hline \multirow[t]{4}{*}{$\theta$} & $(0.2,0.3,0.4)$ & 0.0902 & 9716.1 \\
\hline & $(0.4,0.5,0.6)$ & 0.0901 & 9718.5 \\
\hline & $(0.6,0.7,0.8)$ & 0.0900 & 9720.9 \\
\hline & $(0.8,0.9,1.0)$ & 0.0899 & 9723.3 \\
\hline \multirow[t]{4}{*}{$\mathrm{C}$} & $(20,30,40)$ & 0.0837 & 11063 \\
\hline & $(25,35,45)$ & 0.0784 & 12389 \\
\hline & $(30,40,50)$ & 0.0740 & 13697 \\
\hline & $(35,45,55)$ & 0.0702 & 14990 \\
\hline \multirow[t]{4}{*}{$\mathrm{h}$} & $(3,6,9)$ & 0.0844 & 9996.7 \\
\hline & $(4,7,10)$ & 0.0796 & 10261 \\
\hline & $(5,8,11)$ & 0.0755 & 10511 \\
\hline & $(6,9,12)$ & 0.0721 & 10748 \\
\hline \multirow[t]{4}{*}{$\alpha$} & $(0.3,0.55,0.80)$ & 0.0897 & 9734.8 \\
\hline & $(0.35,0.6,0.85)$ & 0.0892 & 9754.4 \\
\hline & $(0.40,0.65,0.9)$ & 0.0887 & 9773.9 \\
\hline & $(0.45,0.7,0.95)$ & 0.0882 & 9793.3 \\
\hline \multirow[t]{4}{*}{$\lambda$} & $(125,225,325)$ & 0.0942 & 10536 \\
\hline & $(150,250,350)$ & 0.0902 & 11366 \\
\hline & $(175,275,375)$ & 0.0868 & 12189 \\
\hline & $(200,300,400)$ & 0.0837 & 13005 \\
\hline
\end{tabular}

Table 2. Effects of different parameters using graded mean integration method

\begin{tabular}{|c|c|c|c|}
\hline Parameter & & $\mathrm{T}$ & $T C_{G M}(\mathrm{~T})$ \\
\hline \multirow[t]{4}{*}{ A } & $(150,250,350)$ & 0.0969 & 9876.8 \\
\hline & $(200,300,400)$ & 0.1058 & 10306 \\
\hline & $(250,350,450)$ & 0.1139 & 10703 \\
\hline & $(300,400,500)$ & 0.1214 & 11073 \\
\hline \multirow[t]{4}{*}{$\theta$} & $(0.2,0.3,0.4)$ & 0.0869 & 9405.1 \\
\hline & $(0.4,0.5,0.6)$ & 0.0868 & 9407.6 \\
\hline & $(0.6,0.7,0.8)$ & 0.0867 & 9410.2 \\
\hline & $(0.8,0.9,1.0)$ & 0.0866 & 9412.7 \\
\hline \multirow[t]{4}{*}{$\mathrm{C}$} & $(20,30,40)$ & 0.0803 & 10758 \\
\hline & $(25,35,45)$ & 0.0750 & 12087 \\
\hline & $(30,40,50)$ & 0.0706 & 13398 \\
\hline & $(35,45,55)$ & 0.0669 & 14692 \\
\hline \multirow[t]{4}{*}{$\mathrm{h}$} & $(3,6,9)$ & 0.0810 & 9694.0 \\
\hline & $(4,7,10)$ & 0.0761 & 9964.9 \\
\hline & $(5,8,11)$ & 0.0720 & 10220 \\
\hline & $(6,9,12)$ & 0.0685 & 10462 \\
\hline \multirow[t]{4}{*}{$\alpha$} & $(0.3,0.55,0.80)$ & 0.0864 & 9425.1 \\
\hline & $(0.35,0.6,0.85)$ & 0.0859 & 9445.1 \\
\hline & $(0.40,0.65,0.9)$ & 0.0855 & 9464.2 \\
\hline & $(0.45,0.7,0.95)$ & 0.0850 & 9484.1 \\
\hline \multirow[t]{4}{*}{$\lambda$} & $(125,225,325)$ & 0.0984 & 10182 \\
\hline & $(150,250,350)$ & 0.0941 & 11014 \\
\hline & $(175,275,375)$ & 0.0903 & 11838 \\
\hline & $(200,300,400)$ & 0.0870 & 12654 \\
\hline
\end{tabular}

Table 3. Effects of different parameters using signed distance method

\begin{tabular}{|c|c|c|c|}
\hline Parameter & & $\mathrm{T}$ & $T C_{G M}(\mathrm{~T})$ \\
\hline \multirow[t]{4}{*}{$\mathrm{A}$} & $(90,170,250)$ & 0.1415 & 4215.3 \\
\hline & $(100,180,260)$ & 0.1454 & 4285.5 \\
\hline & $(110,190,270)$ & 0.1492 & 4353.8 \\
\hline & $(120,200,280)$ & 0.1529 & 4420.4 \\
\hline \multirow[t]{4}{*}{$\theta$} & $(0.2,0.3,0.4)$ & 0.1374 & 4144.2 \\
\hline & $(0.4,0.5,0.6)$ & 0.1371 & 4146.4 \\
\hline & $(0.6,0.7,0.8)$ & 0.1368 & 4148.5 \\
\hline & $(0.8,0.9,1.0)$ & 0.1366 & 4150.6 \\
\hline \multirow[t]{4}{*}{$\mathrm{C}$} & $(15,25,35)$ & 0.1260 & 4874.2 \\
\hline & $(20,30,40)$ & 0.1171 & 5587.4 \\
\hline & $(25,35,45)$ & 0.1098 & 6286.6 \\
\hline & $(30,40,50)$ & 0.1037 & 6974.4 \\
\hline \multirow[t]{4}{*}{$\mathrm{h}$} & $(3,6,9)$ & 0.1094 & 5784.3 \\
\hline & $(4,7,10)$ & 0.1031 & 5968.6 \\
\hline & $(5,8,11)$ & 0.0978 & 6142.4 \\
\hline & $(6,9,12)$ & 0.0932 & 6307.3 \\
\hline \multirow[t]{4}{*}{$\alpha$} & $(0.25,0.45,0.65)$ & 0.1163 & 6405.2 \\
\hline & $(0.30,0.50,0.70)$ & 0.1155 & 6415.5 \\
\hline & $(0.35,0.55,0.75)$ & 0.1147 & 6425.6 \\
\hline & $(0.40,0.60,0.80)$ & 0.1140 & 6436.3 \\
\hline \multirow[t]{4}{*}{$\lambda$} & $(60,110,160)$ & 0.1127 & 6809.2 \\
\hline & $(70,120,170)$ & 0.1088 & 7218.2 \\
\hline & $(80,130,180)$ & 0.1053 & 7622.2 \\
\hline & $(90,140,190)$ & 0.1021 & 8021.4 \\
\hline \multirow[t]{4}{*}{$\mathrm{m}$} & $(6,11,16)$ & 0.1170 & 6352.0 \\
\hline & $(7,12,17)$ & 0.1170 & 6310.1 \\
\hline & $(8,13,18)$ & 0.1170 & 6268.1 \\
\hline & $(9,14,19)$ & 0.1169 & 6225.5 \\
\hline
\end{tabular}

Table 4. Effects of different parameters using graded mean integration method

\begin{tabular}{|l|l|l|l|}
\hline Parameter & & $\mathrm{T}$ & $T C_{G M}(\mathrm{~T})$ \\
\hline $\mathrm{A}$ & $(90,170,250)$ & 0.1505 & 4025.5 \\
\hline & $(100,180,260)$ & 0.1546 & 4092.0 \\
\hline & $(110,190,270)$ & 0.1586 & 4156.8 \\
\hline & $(120,200,280)$ & 0.1626 & 4220.0 \\
\hline$\theta$ & $(0.2,0.3,0.4)$ & 0.1460 & 3958.2 \\
\hline & $(0.4,0.5,0.6)$ & 0.1457 & 3960.5 \\
\hline & $(0.6,0.7,0.8)$ & 0.1454 & 3962.8 \\
\hline & $(0.8,0.9,1.0)$ & 0.1451 & 3965.0 \\
\hline $\mathrm{C}$ & $(15,25,35)$ & 0.1331 & 4689.2 \\
\hline & $(20,30,40)$ & 0.1231 & 5402.7 \\
\hline & $(25,35,45)$ & 0.1151 & 6105.1 \\
\hline & $(30,40,50)$ & 0.1084 & 6788.4 \\
\hline $\mathrm{h}$ & $(3,6,9)$ & 0.1362 & 4121.0 \\
\hline & $(4,7,10)$ & 0.1281 & 4274.0 \\
\hline & $(5,8,11)$ & 0.1212 & 4418.1 \\
\hline & $(6,9,12)$ & 0.1154 & 4554.6 \\
\hline$\alpha$ & $(0.25,0.45,0.65)$ & 0.1452 & 4643.2 \\
\hline & $(0.30,0.50,0.70)$ & 0.1443 & 4653.0 \\
\hline & $(0.35,0.55,0.75)$ & 0.1433 & 4662.1 \\
\hline & $(0.40,0.60,0.80)$ & 0.1424 & 4671.5 \\
\hline$\lambda$ & $(60,110,160)$ & 0.1406 & 4978.9 \\
\hline
\end{tabular}




\begin{tabular}{|l|l|l|l|}
\hline & $(70,120,170)$ & 0.1356 & 5262.0 \\
\hline & $(80,130,180)$ & 0.1310 & 5539.9 \\
\hline & $(90,140,190)$ & 0.1270 & 5815.0 \\
\hline $\mathrm{m}$ & $(6,11,16)$ & 0.1462 & 5920.7 \\
\hline & $(7,12,17)$ & 0.1463 & 5861.9 \\
\hline & $(8,13,18)$ & 0.1464 & 5803.1 \\
\hline & $(9,14,19)$ & 0.1465 & 5744.3 \\
\hline
\end{tabular}

Case(iii) $T_{d} \leq m \leq T$

Table 5. Effects of different parameters using signed distance method

\begin{tabular}{|c|c|c|c|}
\hline Parameter & & $\mathrm{T}$ & $T C_{G M}(\mathrm{~T})$ \\
\hline \multirow[t]{4}{*}{ A } & $(30,50,70)$ & 0.6811 & 19579 \\
\hline & $(40,60,80)$ & 0.6816 & 19593 \\
\hline & $(50,70,90)$ & 0.6822 & 19622 \\
\hline & $(60,80,100)$ & 0.6827 & 19636 \\
\hline \multirow[t]{4}{*}{$\theta$} & $\begin{array}{ll}(0.12, & 0.22 \\
, 0.32) & \end{array}$ & 0.7321 & 21183 \\
\hline & $\begin{array}{ll}(0.14, & 0.24 \\
, 0.34) & \end{array}$ & 0.7781 & 22695 \\
\hline & $\begin{array}{l}(0.16, \\
0.26,0.36)\end{array}$ & 0.8198 & 24133 \\
\hline & $\begin{array}{l}(0.18, \\
0.28,0.38)\end{array}$ & 0.8578 & 25512 \\
\hline \multirow[t]{4}{*}{$\mathrm{C}$} & $(30,40,50)$ & 0.8201 & 29968 \\
\hline & $(40,50,60)$ & 0.8926 & 39864 \\
\hline & $(50,60,70)$ & 0.9379 & 49577 \\
\hline & $(60,70,80)$ & 0.9689 & 59198 \\
\hline \multirow[t]{4}{*}{$\mathrm{h}$} & $(3,6,9)$ & 0.6403 & 20568 \\
\hline & $(4,7,10)$ & 0.6067 & 21493 \\
\hline & $(5,8,11)$ & 0.5783 & 22363 \\
\hline & $(6,9,12)$ & 0.5537 & 23188 \\
\hline \multirow[t]{4}{*}{$\alpha$} & $(0.25,0.45,0.65)$ & 0.6236 & 18370 \\
\hline & $(0.30,0.50,0.70)$ & 0.5625 & 17012 \\
\hline & $(0.35,0.55,0.75)$ & 0.4951 & 15457 \\
\hline & $(0.40,0.60,0.80)$ & 0.4166 & 13619 \\
\hline \multirow[t]{4}{*}{$\lambda$} & $(60,85,110)$ & 0.6751 & 16426 \\
\hline & $(70,95,120)$ & 0.6707 & 17953 \\
\hline & $(80,105,130)$ & 0.6670 & 19478 \\
\hline & $(90,115,140)$ & 0.6638 & 21001 \\
\hline \multirow[t]{4}{*}{$\mathrm{m}$} & $(6,11,16)$ & 0.7925 & 18475 \\
\hline & $(6.5,11.5,16.5)$ & 0.8500 & 20435 \\
\hline & $(7,12,17)$ & 0.9086 & 22517 \\
\hline & $(7.5,12.5,17.5)$ & 0.9681 & 24722 \\
\hline
\end{tabular}


According to Table 6 , increasing the ordering cost ' $A$ ', $\tilde{C}$, $\tilde{m}, \tilde{\theta}$ results in an increase in the optimal cycle time $T$ and total relevant cost, thus increasing the in $\tilde{\alpha}, \tilde{h}, \tilde{\lambda}$ results in a decrease in the optimal cycle time $T$ and an increase in the total relevant cost.

\section{Conclusion}

When the supplier enables a payment delay connected to order quantity ,in this study we use an fuzzy inventory model for decreasing products to determine the best ordering strategy under inflation. The theoretical repercussion in each case are explained through numerical examples. The variance of different parameters is revealed through sensitivity analysis. A greater buying cost leads to a shorter cycle time and a higher total relevant cost, whereas higher holding cost leads to a shorter cycle time and a higher total relevant cost is obtained. Finally, a higher decay rate leads to a shorter optimal cycle time and a higher total relevant cost. The suggested work can be used to predict demand as a function of stock level time, linear and quadratic demand, selling price, and other factors.

\section{Conflict of interest}

On behalf of all authors, the corresponding author states that there is no conflict of interest

\section{REFERENCES}

[1] D.Dutta, Pavan Kumar, Fuzzy inventory model without shortages using trapezoidal fuzzy number with sensitivity analysis, IOSR Journal of Mathematics, Vol.4, 32- 37, 2015.

[2] Dutta, Pavan Kumar, Optimal policy for an inventory model without shortages consider fuzziness in demand, holding cost and ordering cost, International Journal of Advanced Innovation and Research, Vol.2, No.3, 320-325, 2013.

[3] Goyal,S.K, EOQ under conditions of permissible delay in payments,Journal of Operational Research Society, Vol.36, 335338,1985 .

[4] Goyal,S.K, Giri,B.C, Recent trends in modelling of deteriorating inventory, European Journal of Operational Research, Vol.134, 1-16, 2001.

[5] Harish Nagar, Priyanka Surana, Fuzzy inventory model for deteriorating items with fluctuating demand and using inventory parameters as Pentagonal Fuzzy numbers, Journal of computer and Mathematical Science, Vol.6, No.2, 55-65, 2015.

[6] Huey-Ming Lee, Jing-Shing Yao, Economic order quantity in Fuzzy sense for Inventory without Back order Fuzzy sets,Journal of Information and Engineering, Vol.21, 673-694, 2005 .

[7] S.K.Indirajit Sinha, P.N.Samantha, U.K.Mishra, Fuzzy Inventory Model with Shortages under Full Backlogged Using Signed Distance Method,International Journal for research in Applied
Science and Engineering Technology, Vol.4, No.1, 197-203, 2016.

[8] Jaggi C.K, Pareek S, Khanna A, Nidhi, Optimal Replenishment policy for fuzzy Inventory model with Deteriorating items and under allowable shortages under Inflationary conditions, Yugoslav Journal of Operations Research, Vol.10, 2298/YJOR150202002Y, 2016.

[9] Jershan Chiang-Shing S Yao, huey M Lee, Economic reorder point for fuzzy backorder quantity, European Journal of Operation Research, Vol.109, 183-202, 1998.

[10] Khanna,S, Ghosh, S.K, and Chaudhuri, K.S, An EOQ model for a deteriorating item with time-dependent quadratic demand under permissible delay in payment. Applied Mathematics and Computation, Vol.218, 1-9, 2011.

[11] Kumar,S, Rajput ,U.S, Fuzzy Inventory model for Deteriorating items with Time dependent demand and partial backlogging, Applied Mathematics, Vol.6, 496- 509, 2015.

[12] G.Michael Rosario, R.M.Rajalakshmi, A Fuzzy Inventory model with allowable shortage using different fuzzy numbers, International Journal of computational and Applied Mathematics, Vol.12, No.1, 227-240, 2017.

[13] Sana, S.S, Chaudhuri, K.S, A deterministic EOQ model with delays in payments and price discount offers. European Journal of Operations Research, Vol.184, 509-533, 2008.

[14] Shinn, S.W., Hwang, H.P., Sung, S.,Joint price and lot size determination under condition of permissible delay in payments and quantity discounts for freight cost, European Journal of Operational Research, Vol.91, 528-542, 1996.

[15] Taheri-Tolgari, J, Mirzazadeh, A, Jolai, F., An inventory model for imperfect items under inflationary conditions with considering inspection errors, Computers and Mathematics with Applications, Vol.63, 1007-1019, 2012.

[16] Teng, J.T. Yang, H.L, Chern, M.S, An inventory model for increasing demand under two levels of trade credit linked to order quantity. Applied Mathematical Modelling, Vol.37, 2624-2632, 2013.

[17] Tripathi, R.P, Inventory model with time-dependent demand rate under inflation when supplier credit linked to order quantity. International Journal of Business and Information Technology, Vol.1, No.3, 174-183, 2011.

[18] Tripathi, R.P., Dinesh Singh, Mishra.T, An EOQ model for deteriorating Items with Exponential Time- Dependent Demand Rate under Inflation when supplier credit is linked to order quantity. International Journal of Supply and Operations Management, Vol.1, No.1, 20-37, 2014.

[19] Tripathi, R.P, Pandey, H.S, An EOQ model for deteriorating Items with Weibull Time- Dependent Demand Rate under Trade Credits. International Journal of Information and Management Sciences, Vol.24, No.4, 329-347, 2013.

[20] Ouyang, L.Y, Chang, C.T, Optimal production lot with imperfect production process under permissible delay in payment and complete backlogging. International Journal of Production Economics, Vol.144, 610-617, 2013. 